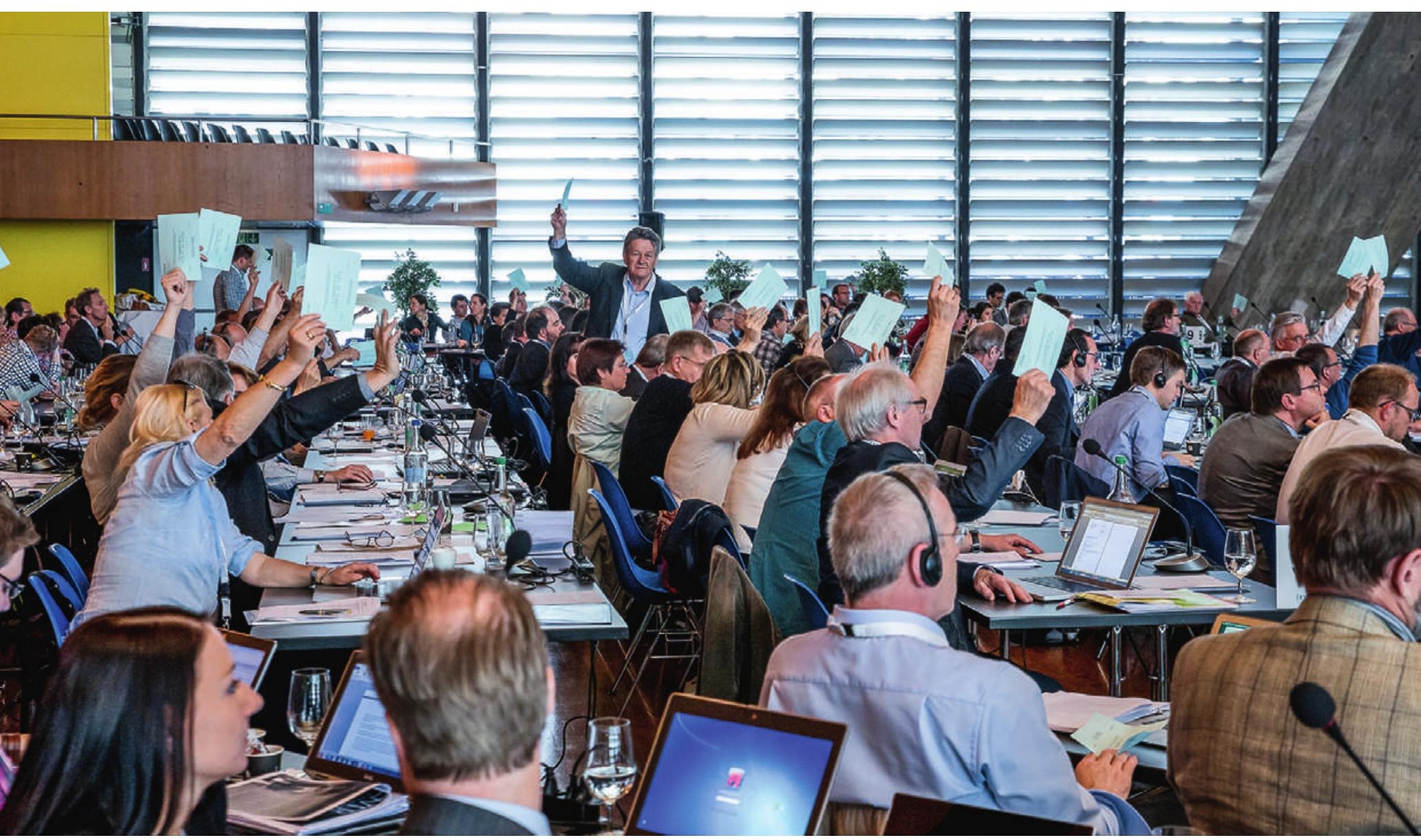

\title{
Protokoll der ersten Ärztekammer im Jahr 2015
}

\section{Monika Henzen}

Leiterin Zentrales Sekretariat

\section{Begrüssung, Mitteilungen, Bestellung des Büros}

Jürg Schlup/Präsident FMH begrüsst die Delegierten zur heutigen Ärztekammer. Als Gäste heisst er Joachim Eder/Ständerat ZG, Hans-Rudolf Koelz/Vizepräsident SIWF, Jean-Pierre Keller/Vizepräsident SIWF, Beat Bär/ Geschäftsführer FMH Services, Bruno Kesseli/Chefredaktor SÄZ, Ruedi Bienz/Geschäftsführer EMH sowie Nicole Beutler/FurrerHugi Partner herzlich willkommen.

Zu den Traktanden 3 werden als Gäste Roland Burger/ Revisionsfirma BDO, zu 5.3.1 Michel Meier/Rechtsberater AeG Solothurn, zu 5.3.2 und 5.3.3 Prof. Dr. med. Christian
Kind/Präsident der Zentralen Ethikkommission der SAMW sowie zu 8 Dr. Walter Gratzer und Marc Widmer/ KPMG erwartet.

Anne-Geneviève Bütikofer/GS FMH gibt die üblichen organisatorischen Mitteilungen und verweist auf die heute zusätzlich verteilten Sitzungsunterlagen. Die Delegierten haben ebenfalls Gelegenheit, mittels Fragebogen ihr Feedback zum neuen Ablagesystem der Sitzungsunterlagen abzugeben.

Anschliessend wird das Büro der ÄK bestellt. Dieses besteht aus dem Präsidenten, der Generalsekretärin und den nachfolgenden Stimmenzählenden:

Christian Bernath, Hans-Anton Vogel, Philippe Rheiner, Marcel Stampfli, Hans Ulrich Iselin, Daniel Acker- 


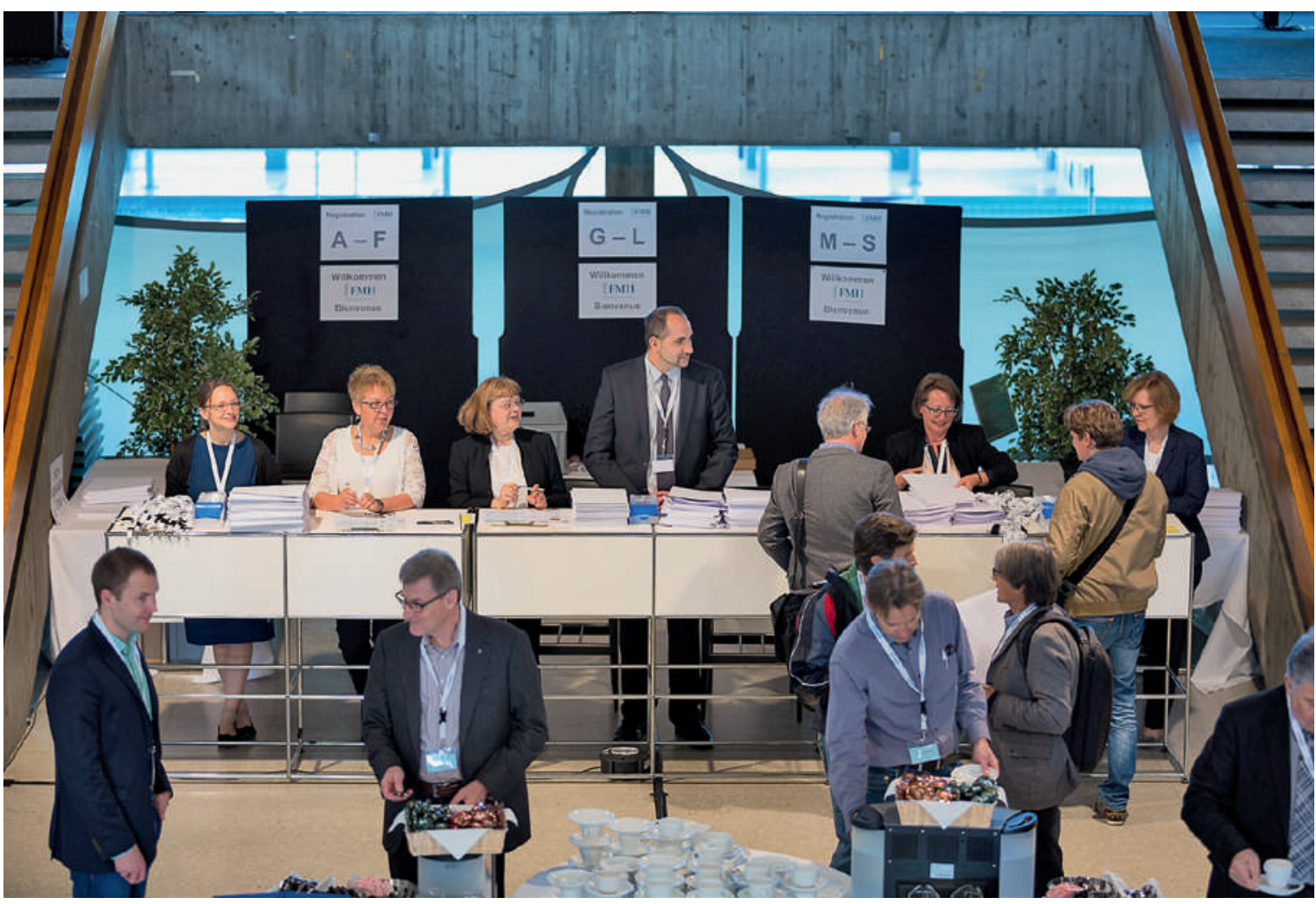

Die Delegierten der Kantonal- und Fachgesellschaften sowie Dachverbände erhalten ihre Stimmkarten und Unterlagen von den FMH-Mitarbeitenden am Empfang.

mann, Hans-Jakob Riedtmann, Robert Weber, Hervé Spechbach, Daniel Schröpfer.

Die Stimmenzählenden werden ohne Gegenstimme gewählt.

\section{Traktandenliste}

Die Traktandenliste wird einstimmig genehmigt.

Der Präsident beantragt, die ÄK spätestens um 17.45 Uhr zu beenden. Da ein Antrag auf Festlegung des Tagungsendes auf 17.00 Uhr eingereicht wird, stimmen die Delegierten über die beiden gegenübergestellten Anträge ab.

\section{Beschluss:}

Der Antrag Festlegung des Tagungsendes auf 17.45 Uhr wird mit 63 Ja zu 28 Nein gutgeheissen.

Gemäss Art. 11 Abs. 3 der GO legt Jürg Schlup/Präsident FMH fest, dass ab 17.45 Uhr keine Beschlüsse mehr gefasst und keine Wahlen mehr vollzogen werden.

\section{Eingangsreferat Präsident FMH}

Jürg Schlup/Präsident FMH eröffnet die heutige ÄK, die ohne den langjährigen Vizepräsidenten Ernst Gähler stattfindet. Unverhofft und ohne Abschied wurde er am 12.3.2015 mitten aus dem Leben gerissen. Herausgerissen aus seinem Wirken als Arzt, Ehepartner, Familienvater, Grossvater, Kantonsrat und Vizepräsident der
FMH. Der Präsident erinnert in seinen Worten an eine aussergewöhnliche und prägende Persönlichkeit, die auch in schwierigen Situationen das Ziel nicht aus den Augen verloren und dafür gekämpft hat. Die FMH verliert mit Ernst Gähler einen engagierten Arzt, Freund und Kollegen. Sein Tod hinterlässt eine grosse Lücke.

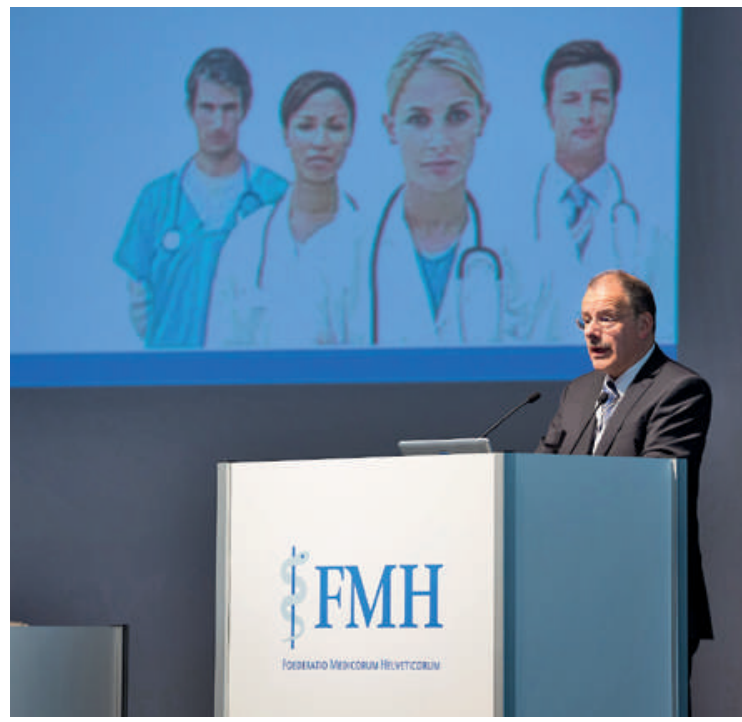

FMH-Präsident Dr. med. Jürg Schlup heisst die 200 Delegierten der Ärztekammer - das «Parlament» der FMH -

herzlich willkommen. 
Hans-Anton Vogel/AR nimmt mit eindrucksvollen appenzellischen Impressionen Abschied von Ernst. Er würdigt ihn als Menschen und Freund, dessen Handeln von Zuversicht, Grosszügigkeit und Hoffnung geprägt war. Ernst hat es immer verstanden, die wortkargen Appenzeller für sich zu gewinnen. Er hat ihre Töne und Stimmungen stets gefunden. Das Wesen des Appenzellers mag rau und herb sein, aber es ist immer echt und klar. So echt und klar waren auch seine Ziele. Ernst war mit dem Appenzellerland stark verwurzelt. Die Liebe zur Natur, zur Landschaft und zur Kultur war ihm wertvoll. Daraus hat er immer jene Kraft geschöpft, die er brauchte, um seine Ziele zu verfolgen. Gleich der Unerschöpflichkeit des Wassers ist er mit seiner Arbeitskraft umgegangen und hat beharrlich, gemäss seinem Motto «Nöd logg lo gwönnt», die Herausforderungen angepackt. Was er erarbeitet und angedacht hat, strahlt ins ganze Land. Und sein Tod führt uns, wie es bei Shakespeare heisst, vor Augen: Auch du bist Gott einen Tod schuldig.

Jürg Schlup/Präsident FMH dankt dem Verstorbenen im Namen der Ärztinnen und Ärzte für den grossen und ausserordentlichen Einsatz zum Wohle der Patienten und der Ärzteschaft. Für ihn verfügte Ernst über jene vier Qualitäten, die für eine tragfähige und gute Beziehung zwischen Patient und Arzt entscheidend sind: gegenseitige Achtung, liebevolles Verstehen und Handeln, Bemühen um Sachlichkeit sowie Bescheidenheit. Er würde auch heute dazu ermuntern, gemeinsam für gute Lösungen zu kämpfen, getreu seinem Leitsatz «Mitenand got's besser und nöd logg lo gwönnt.»

Die Delegierten gedenken in einer Schweigeminute des verstorbenen Vizepräsidenten.

Das Werk von Ernst soll weitergeführt werden und der ZV hat die von ihm betreuten Dossiers unter sich aufgeteilt. Das Dossier "Ambulante Tarife» und «Tarifrevision» wird mehrheitlich Urs Stoffel/ZV FMH übertragen. Remo Osterwalder/ZV FMH übernimmt ebenfalls einen Teil des Dossiers «Ambulante Tarife». Gert Printzen/ZV $F M H$ ist für den Bereich "Paramedizinische Berufe» und der Präsident für die Vertragsverhandlungen im ambulanten Bereich zuständig.

Der Präsident dankt den ZV-Mitgliedern wie auch dem Team «Ambulante Tarife», unter der Leitung von Kerstin Schutz, bestens für das grosse und ausserordentliche Engagement in dieser schwierigen Zeit.

Was hat die FMH ausserdem in den vergangenen Monaten bewegt? Für Jürg Schlup/Präsident FMH bleibt die Tarifrevision nach wie vor eine grosse Herausforderung. 2013 hat NR Humbel den Bundesrat mit einer Interpellation zum Thema «Leistungsgerechte Korrekturen am Arzttarif TARMED» (13.3223) befragt: Wie und in welchem Zeitrahmen gedenkt der Bundesrat von sei-

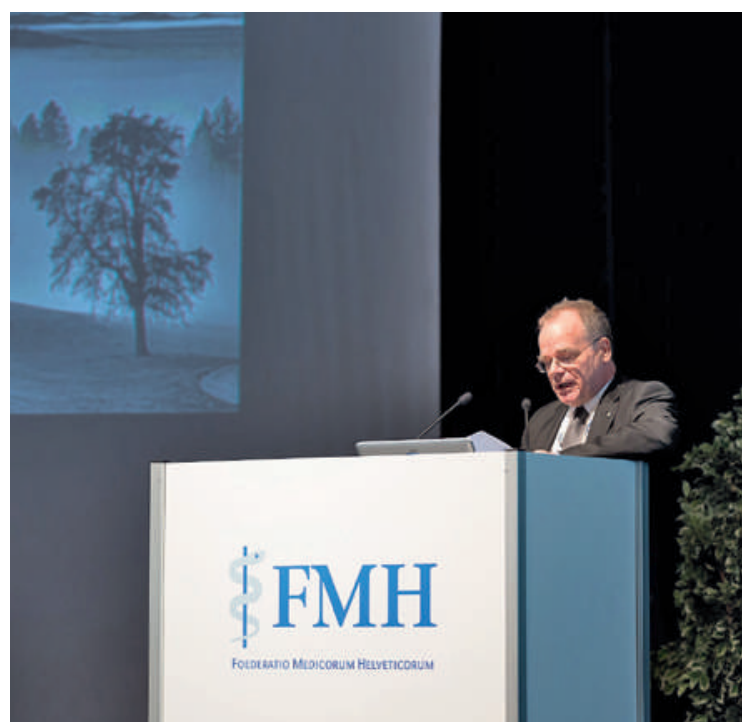

Der Präsident der Appenzellischen Ärztegesellschaft Dr. med. Hans-Anton Vogel hält die Trauerrede für den verstorbenen FMH-Vizepräsidenten und seinen langjährigen Weggefährten, Dr. med. Ernst Gähler.

ner Kompetenz, TARMED-Tarife anzupassen, Gebrauch zu machen? Der Bundesrat antwortete: «Daher wird der Bundesrat in einer ersten Phase die Wahrnehmung seiner neuen Kompetenz im Sinne von punktuellen Anpassungen bezogen auf ambulant tätige Hausärzte in freier Praxis prüfen und gegebenenfalls 2014 umsetzen. Anpassungen von ganzen Leistungsbereichen im TARMED sind wesentlich aufwendiger und kommen daher erst in einer zweiten Phase, voraussichtlich 2015, in Frage.» Der Bundesrat hat also von zwei Tarifeingriffen gesprochen. Damit stehen heute zwei Szenarien auf dem Spiel. Das Modell der FMH sieht die Gestaltung und Weiterentwicklung durch die Tarifpartner vor. Der Bundesrat jedoch tönt in seiner Antwort an NR Humbel einen möglichen zweiten Tarifeingriff an. Für den ZV ist klar, welches Modell weiterzuverfolgen ist. Zusätzliche Informationen über den aktuellen Stand werden die Delegierten heute ebenfalls unter Traktandum 9 erhalten.

Die FMH wird in der Öffentlichkeit als wichtige Partnerin wahrgenommen und will ihren Einfluss verstärken. Im Rahmen des laufenden Strategieprozesses hat die FMH 2014 eine kleine Umfrage einerseits bei Ärztinnen und Ärzten und anderseits bei Politikern und Medienschaffenden durchgeführt. Die FMH wollte wissen, wie sie in der Öffentlichkeit und bei der Ärzteschaft wahrgenommen wird. Die Befragten konnten aus einem Dutzend Adjektiven auswählen, dabei ergab sich bei drei der vier meistgenannten Charakteristika eine erstaunliche Übereinstimmung: Beide befragten Gruppen beschrieben die FMH am häufigsten mit kompetent, professionell und glaubwürdig. Eine grosse 
Wahrnehmungsdifferenz ergab sich zwischen beiden Gruppen einzig beim politischen Einfluss. Während die Ärzteschaft die FMH als wenig einflussreich einstufte, beurteilten die Politiker und Medienschaffenden die FMH als sehr einflussreich. Die FMH wird als wichtige Partnerin bei den Herausforderungen im Gesundheitswesen wie bei der politischen Meinungsbildung wieder vermehrt einbezogen. Die Stellungnahmen werden nicht nur gelesen, sondern auch im Parlament namentlich diskutiert, wie dies in den Ratsprotokollen der vergangenen Frühjahrs- und Wintersession ersichtlich wird und aufgrund der zunehmend häufigen Einladungen zu parlamentarischen Anhörungen hervorgeht. Die FMH ist präsent und nimmt Einfluss. Diese externe Beurteilung ist erfreulich. Unsere internen Meinungsdifferenzen und der fehlende Konsens werden allerdings ebenso wahrgenommen, wie die Umfrage zeigt. Jürg Schlup/Präsident $F M H$ wünscht sich eine FMH, die sich entwickelt und sich den Veränderungen stellt. Er wünscht sich Menschen, die Freude an der Zukunft haben und weniger an der Vergangenheit; die sich für sachliche und umsetzbare Lösungen interessieren.

\section{Jahresberichte 2014}

\section{Mitteilungen des Präsidenten SIWF}

Für Werner Bauer/Präsident SIWF ist es wichtig, dass die Weiter- und Fortbildung in der ÄK den nötigen Rückhalt hat. Wenn die Weiter- und Fortbildung in ärztlichen Händen bleiben soll, muss die FMH auch die Verantwortung dafür übernehmen. Er nutzt die Gelegenheit, Informationen zu aktuellen Projekten und Schwerpunktthemen zu geben. Die Strukturveränderungen sowohl im stationären wie ambulanten Bereich gehen rasant weiter und haben Einfluss auf die zeitlichen und finanziellen Ressourcen. Die Weiterbildung ist nach wie vor nicht wirklich gesichert, weshalb ein grösserer Einsatz seitens der Weiterbildner wichtig ist. Der Stellenwert des Lehrens und Lernens in den Spitälern ist konfrontiert mit dem Stellenwert der Dienstleistung und der Ökonomie. Das SIWF versucht, die Weiterbildenden zu stärken und zu motivieren, neue Projekte und Initiativen zu entwickeln. Diese Aktivitäten kosten Geld und wirken sich entsprechend auf die Jahresrechnung und das Budget aus. Die aktuelle Jahresrechnung 2014 ist jedoch kein Zeichen einer schlechten Haushaltsführung, sondern vielmehr Ausdruck der Verantwortung, die für die Zukunft der Weiter- und Fortbildung entscheidend und prägend ist. Eine Institution gewinnt dann weiter an Qualität, wenn sie in die Weiterbildung investiert. Zur Zeit berei- tet das Bundesamt für Gesundheit die Akkreditierung 2018 aller Weiterbildungsgänge vor. Alle sieben Jahre entwickelt sich dieser Akkreditierungsprozess, der für das SIWF und die Fachgesellschaften mit einem administrativen Aufwand verbunden ist. Am 23.9.2015 findet zum zweiten Mal das Medical Education Symposium in Bern statt. Dieses ist den Perspektiven der Weiterbildung gewidmet. Alle Interessierten sind herzlich eingeladen, daran teilzunehmen.

\subsection{Jahresbericht des SIWF}

Christoph Hänggeli/Geschäftsführer SIWF informiert über den Geschäftsbericht des SIWF, der mit einer Auflage von 4500 Exemplaren u.a. auch an alle Leiter der anerkannten Weiterbildungsstätten abgegeben wurde. Bei drei Themen, die das Jahr 2014 wesentlich geprägt haben, sind inzwischen wichtige Meilensteine erreicht worden.

Am 20.3.2015 verabschiedeten die eidgenössischen Räte die Revision des Medizinalberufegesetzes (MedBG). Fünf Jahre harte Überzeugungsarbeit mit unzähligen Sitzungen und Stellungnahmen waren notwendig, um die wichtigen Anliegen der Ärzteschaft sowie der Patientinnen und Patienten einzubringen. Besonders umkämpft war die Frage, ob Ärzte überhaupt eine Landessprache sprechen müssen. Erst in der finalen Einigungskonferenz haben sich National- und Ständerat zu einer befriedigenden Lösung durchgerungen. Noch wichtiger als die Sprachkompetenz war die Forderung nach einem vollständigen Ärzteregister. Hier ist nun sichergestellt, dass in Zukunft alle ausländischen Arztdiplome amtlich überprüft werden, bevor ein Arzt bzw. eine Ärztin eine Tätigkeit in einem Spital antritt. Der Bundesrat wird nun die Ausführungsbestimmungen erlassen. Mit der Inkraftsetzung ist voraussichtlich $2016 \mathrm{zu}$ rechnen.

Die Schweizerische Konferenz der kantonalen Gesundheitsdirektorinnen und -direktoren (GDK) hat im November 2015 die interkantonale Vereinbarung über die Finanzierung der ärztlichen Weiterbildung gutgeheissen. Die Kantone müssen einerseits den anerkannten Weiterbildungsstätten im Minimum CHF 15000 pro Assistenzarzt und Assistenzärztin an die strukturierte Weiterbildung bezahlen. Anderseits gibt es neu einen interkantonalen Ausgleich zwischen Kantonen, die viele Assistenzärzte beschäftigen, gegenüber jenen Kantonen, die wenig weiterbilden. Die Zustimmung von 18 Kantonen ist für die Ratifizierung des Konkordates ist notwendig.

Das e-Logbuch umfasst aktuell 40 eidgenössische Facharzttitel und 16 Schwerpunkte. 96\% aller Weiterbildungsperioden können im e-Logbuch dokumentiert werden. Von den 10000 Assistenzärzten sind 7500 registrierte 


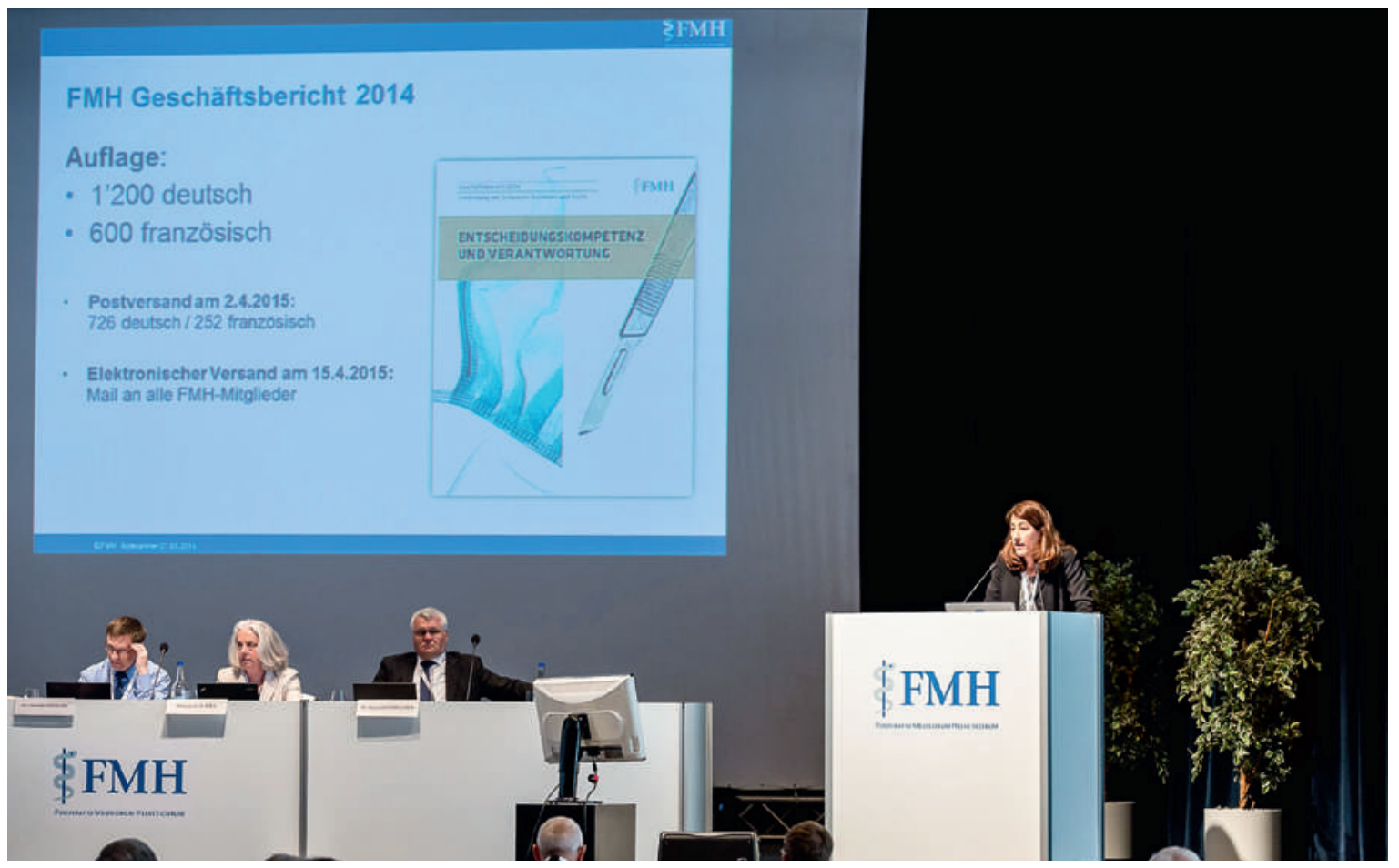

Anne-Geneviève Bütikofer, Generalsekretärin der FMH, stellt den Geschäftsbericht 2014 vor, der in gedruckter und elektronischer Form veröffentlicht wird.

Benutzer. Ziel ist die Aufschaltung aller Fachgebiete bis Mitte 2015. Auch das Cockpit für die Geschäftsstelle SIWF und die Titelgesuche können so direkt über das eLogbuch eingegeben werden. Die Daten müssen nicht mehr auf ein zweites System übertragen werden.

Der Jahresbericht SIWF ist unter www.siwf.ch als e-Paper abrufbar.

Antrag:

Die Geschäftsleitung SIWF beantragt der ÄK, den Jahresbericht 2014 des SIWF zu genehmigen.

\section{Beschluss:}

Der Jahresbericht SIWF wird einstimmig genehmigt.

\subsection{Jahresbericht der FMH}

Anne-Geneviève Bütikofer/GS FMH präsentiert den Jahresbericht 2014 der FMH, welcher mit einem neuen Bildkonzept erscheint. Dieser wurde am 2.4.2015 in Papierform und am 15.4.2015 elektronisch zugestellt. Der Jahresbericht war 2014 dem Leitthema «Entscheidungskompetenz und Verantwortung» gewidmet. Eine Diskussion zu diesem Thema fand mit NR Dr. med. Yvonne Gilli und Dr. med. Adrian Wirthner statt. Es zeigte sich, dass Entscheidungskompetenz und Verantwortung bei vielen aktuellen und substantiellen Themen der Ärzteschaft prägende Faktoren und Werte sind. Dem Jahresbericht sind weiter die FMH-Ärztestatistik sowie eine Übersicht der FMH-Dienstleistun- gen beigelegt. Diese sind auch online verfügbar. Die Berichte des ZV, der GS und der Abteilungen dokumentieren die politischen, strategischen und operativen Haupttätigkeiten. Neu erscheint ein Bericht über die internationalen Mandate.

Der Jahresbericht FMH umfasst eine Auflage von 1200 dt. und 600 frz. Weitere Informationen können unter www.fmh.ch oder via APP entnommen werden.

Jürg Schlup/Präsident FMH dankt der Generalsekretärin im Namen des ZV für die anspruchsvolle Arbeit, die sie hochmotiviert täglich für die FMH leistet.

\section{Antrag:}

Der ZV FMH beantragt der ÄK, den Jahresbericht 2014 der FMH zu genehmigen.

\section{Beschluss:}

Der Jahresbericht der FMH wird mit 101 Ja und 2 Enthaltungen genehmigt.

\section{Jahresrechnungen 2014}

\subsection{Jahresrechnung des SIWF}

Christoph Hänggeli/Geschäftsführer SIWF konnte in den letzten 10 Jahren jeweils ein positives Ergebnis präsentieren, womit es dem SIWF gelang, Reserven in der Höhe von CHF 6 Mio. zu akkumulieren. Inzwischen hat das SIWF für die Weiterbildung relevante Projekte entwickelt, um die Reserven zielgerichtet abzubauen 
bzw. sinnvoll zu investieren. Dementsprechend schliesst die Jahresrechnung 2014 nun mit einem Verlust von CHF 889000 ab. Diese Entwicklung wurde bereits im letzten Jahr bei der Präsentation der Rechnung 2013 angekündigt. Das Jahr 2013 markiert nun definitiv einen Wendepunkt, indem in den nächsten Jahren die Ausgaben bei unveränderten Rahmenbedingungen die Einnahmen übersteigen werden. Die Abweichung zum budgetierten Verlust von CHF 287000 ist hauptsächlich dem e-Logbuch zuzuschreiben, das wegen der Komplexität des Projektes wesentlich höhere Kosten verursacht hat als prognostiziert. Auch weitere Projekte sowie die internen Verrechnungen gegenüber der FMH fielen höher aus als budgetiert. Ein Missverhältnis zwischen Einnahmen und Ausgaben existiert insbesondere bei den Einsprachekommissionen und im Bereich Fortbildung. Hier besteht Handlungsbedarf. Für die Einnahmen ist vorab die Zahl der erteilten Facharzttitel massgebend. 2014 wurden 1500 Facharzttitel erteilt. Bemerkenswert ist, dass der Anteil der ausländischen Ärzte, die einen eidgenössischen Titel erwerben, inzwischen auf $44 \%$ angestiegen ist. Im Hinblick auf das Budget 2016 und die Finanzplanung 2017/18 wird das SIWF prüfen, welche Massnahmen ergriffen werden müssen, um die Einnahmen und Ausgaben längerfristig ins Lot zu bringen.

\subsection{Jahresrechnung der FMH}

Zu diesem Traktandum wird ebenfalls Roland Burger/ $B D O$ begrüsst. Emanuel Waeber/Leiter Verwaltung und Finanzen FMH erläutert die Jahresrechnung 2014, welche mit einem Gewinn von CHF 1,308 Mio. abschliesst. Dieser Gewinn resultiert auf der Empfehlung der Revisionsgesellschaft, die stillen Reserven des Arbeitgeberbeitrags in der Höhe von CHF 634000 zugunsten der PAT BVG (Pensionskasse) aufzulösen. Ohne diese Auflösung wäre das Resultat mit einem Gewinn in der Höhe von CHF 674000 ausgefallen. Das positive Resultat ist auch darauf zurückzuführen, dass budgetierte Projekte im Betrag von CHF 730000 nicht realisiert wurden. Der Ertrag ist gegenüber dem Vorjahr jedoch um CHF 2,54 Mio. höher ausgefallen. Andererseits ist der Aufwand gegenüber dem Vorjahr um CHF 1,93 Mio. gestiegen. Die Einnahmen und Ausgaben sind jedoch im Auge zu behalten und die von der ÄK am 30.10.2014 beschlossene Ausgabenbremse ab Budget 2016 ist weiter zu verfolgen.

Das Umlaufvermögen beträgt CHF 17,6 Mio., was 73\% der Bilanzsumme entspricht. Die kurzfristigen Verbindlichkeiten mit Fremdkapital sind bedeutend tiefer als 2013. Der Grund liegt darin, dass die kurzfristigen Verbindlichkeiten gegenüber Dritten um CHF 1,4 Mio. tiefer ausgefallen sind. Der Betriebsertrag ist gegen-

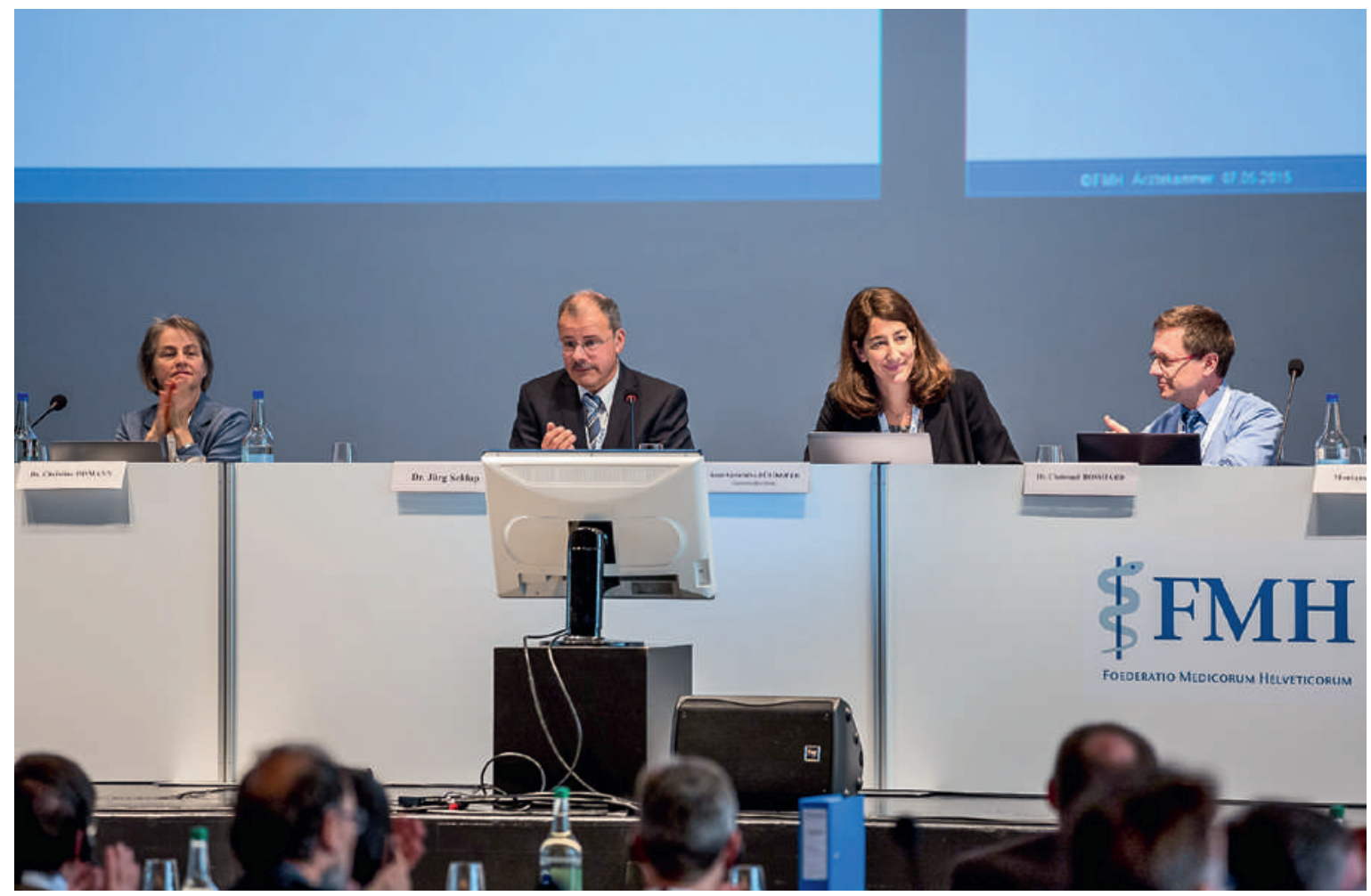

Konzentriert und gleichzeitig schwungvoll führt die FMH-Führungscrew mit Präsident Jürg Schlup (2. von links), Generalsekretärin Anne-Geneviève Bütikofer (2. von rechts) und den Zentralvorstandsmitgliedern durch die dicht befrachtete Traktandenliste. 
über dem Vorjahr um CHF 2,5 Mio. höher. Die Eigenkapitalquote beträgt weiterhin $59 \%$.

Der ZV schlägt vor, den Unternehmensgewinn bzw. -verlust wie folgt aufzuteilen: CHF 1,308 Mio. wird an das Kapital der FMH, der Verlust des SIWF an die Reserve des SIWF zugewiesen.

\subsection{Bericht der Geschäftsprüfungskommission} Adrian Sury/Präsident GPK rapportiert über die Aktivitäten der GPK, welche in gleicher Zusammensetzung getagt hat. Das Berichtsjahr 2014 war geprägt von der finanziellen Zukunft der FMH, die aufgrund der immer grösser werdenden Schere zwischen Einnahmen und Ausgaben mit strukturellem Defizit innert drei Jahren kritisch werden wird. Aus diesem Grunde hat die GPK an der ÄK vom 30.10.2014 den Antrag zur Schaffung von einem operativen finanziellen Spielraum eingereicht, der von der ÄK im Inhalt mit der Schaffung einer Taskforce gutgeheissen wurde.

Die GPK hat am 6.6.2014 die Abschaffung der Wiedereinstiegshilfe beschlossen. Das neue Reglement gilt jedoch erst für die neu gewählten Mitglieder des ZV sowie des SIWF. Die heute Gewählten unterstehen weiterhin dem alten Reglement (Besitzstandswahrung). Die GPK hat bereits 2013 vorgeschlagen, das IPI in das Departement eHealth zu integrieren. Die ÄK wird am 29.10.2015 weiterführende Entscheide fällen. Für die HPC-Karte zeichnet sich eine neue und kostengünstigere Lösung ab. Weitere Baustellen, die von der GPK beobachtet werden, sind das Risikomanagement (neues Rechnungslegungsrecht in Zusammenarbeit mit der Revisionsstelle), das Personalreglement, das überarbeitet und 2015 eingeführt wurde, das Anlagereglement, E-Governance und die Kommunikationsstrategie. Das an der ÄK vom 30.10.2014 beschlossene neue Sitzungs- und Spesenentschädigungsreglement für FMH-Organe und -Kommissionen wird bereits umgesetzt. Die Offenlegung der EMH-Geschäftszahlen konnte mittlerweile ebenso erreicht werden.

Das positive Ergebnis der Jahresrechnung ist hauptsächlich auf die Nichtrealisierung verschiedener Projekte sowie die Auflösung der stillen Reserven zurückzuführen. Die Jahresrechnung 2014 zeigt jedoch, dass nach wie vor strukturelle finanzielle Probleme vorliegen.

Die GPK hat an der Abschlussbesprechung vom 11.3.2015 der Erfolgsrechnung und Bilanz 2014 teilgenommen und vom Ergebnis der konsolidierten Rechnung und der korrekten Rechnungsführung 2014 Kenntnis genommen. Die GPK unterstreicht die konstruktive Gesprächskultur beim ZV und beim SIWF und die ausgezeichnete Arbeit. Sie beantragt, die Jahresrechnung 2014 der FMH und des SIWF zu genehmigen und Décharge zu erteilen. Roland Burger/BDO präsentiert in Kurzform den Revi- sionsbericht FMH. Die Revisionsstelle hat die Jahresrechnung der FMH per Ende 2014 geprüft. Nach ihrer Beurteilung entspricht die Jahresrechnung für das am 31.12.2014 abgeschlossene Geschäftsjahr dem schweizerischen Gesetz und den Statuten der FMH. Im Namen der BDO bedankt er sich beim ZV für das entgegengebrachte Vertrauen und die gute Zusammenarbeit mit dem GS FMH.

Da es zu den Jahresrechnungen SIWF und FMH keine Wortmeldungen und Fragen gibt, wird über die Genehmigung abgestimmt.

\subsection{Genehmigung Jahresrechnung des SIWF}

Antrag:

Die Geschäftsleitung SIWF beantragt der ÄK, die Jahresrechnung 2014 des SIWF zu genehmigen.

Beschluss:

Die Jahresrechnung SIWF wird mit 101 Ja und 4 Enthaltungen genehmigt.

\subsection{Genehmigung Jahresrechnung der FMH}

\section{Antrag:}

Der ZV FMH beantragt der ÄK, die Jahresrechnung 2014 der FMH zu genehmigen.

\section{Beschluss:}

Die Jahresrechnung der FMH wird mit 101 Ja und 7 Enthaltungen genehmigt.

\section{Déchargen-Erteilungen}

Der Vorstand SIWF und der ZV FMH beantragen der ÄK, den Empfehlungen der GPK sowie der Kontrollstelle zu folgen und ihnen für das Geschäftsjahr 2014 Décharge zu erteilen.

4.1 Décharge-Erteilung SIWF

Antrag:

Der Vorstand SIWF beantragt der ÄK, den Empfehlungen der Kontrollstelle sowie der GPK zu folgen und dem Vorstand SIWF für das Geschäftsjahr 2014 Décharge zu erteilen.

Beschluss:

Die ÄK erteilt dem Vorstand SIWF einstimmig Décharge für das Geschäftsjahr 2014

\subsection{Décharge-Erteilung Zentralvorstand FMH \\ Antrag:}

Der ZV FMH beantragt der ÄK, den Empfehlungen der Kontrollstelle sowie der GPK zu folgen und den Mitgliedern des ZV für das Geschäftsjahr 2014 Décharge zu erteilen.

Beschluss:

Die ÄK erteilt dem ZV FMH einstimmig Décharge für das Geschäftsjahr 2014. 


\section{5. Änderungen in Statuten, Geschäfts- ordnung und Standesordnung}

\subsection{Anpassung der FMH-Statuten}

5.1.1 Antrag des Berufsverbandes der Haus- und Kinderärztinnen Schweiz (MFE) auf Anerkennung als mitspracheberechtigte Organisation in der Ärztekammer (gemäss Anhang III)

Der Antrag wurde vom Berufsverband der Haus- und Kinderärztinnen Schweiz (MFE) zwischenzeitlich zurückgezogen.

5.1.2 Anerkennung von neuen Fachgesellschaften und Einsitz in der Ärztekammer (gemäss Anhang II) - Fachgesellschaft für Gefässchirurgie und Fachgesellschaft für Thoraxchirurgie Die beiden früheren Schwerpunkte "Gefässchirurgie» und «Thoraxchirurgie» wurden Anfang 2015 neu zu Facharzttiteln und müssen somit formell durch die ÄK anerkannt werden. Werner Bauer/Präsident SIWF erläutert, dass gemäss Art. 18 und Art. 33 der Statuten der FMH jede Facharztgesellschaft Anrecht auf einen Sitz in der ÄK hat. 58 Sitze sind für die Fachgesellschaften reserviert.

Antrag:

Anerkennung der beiden neuen Fachgesellschaften durch die ÄK und Einsitz in die ÄK. Beschluss:
Dem Antrag wird mit 136 Ja und 2 Nein zugestimmt.

\subsection{Ergänzung der FMH-Geschäftsordnung}

\subsubsection{Rekursfristen bei Ausschluss gemäss Art. 9} Abs. 4 und 5 Statuten; neuer Art. 3bis

Bei der Behandlung eines Rekurses gegen den Ausschluss eines Mitglieds einer Basisorganisation wurde offensichtlich, dass weder die Statuten noch die Reglemente der FMH eine Frist vorsehen, innerhalb der gegen einen Entscheid über den Ausschluss eines Mitglieds Rekurs eingelegt werden kann (mit Ausnahme der Verfahren im Zusammenhang mit den Standesverfahren). Der ZV möchte diese Lücke schliessen und schlägt der ÄK vor, in der GO eine Rekursfrist von 30 Tagen einzuführen. Andreas Haefeli/AG interessiert, ob die Regelung betreffend Frist während den Gerichtsferien klar ist.

Hanspeter Kuhn/Leiter Rechtsdienst FMH hält fest, dass diese Fragen nach den Statuten im übergeordneten Zivilrecht geregelt sind; es ist nicht notwendig, diese Regeln hier im konkreten Anwendungsfall nochmals wiederzugeben.

Für Hans-Jakob Rietmann/AGZ ist unklar, ob die Rekursfrist von 30 Tagen ab Versand oder Empfang beim Mitglied zu laufen beginnt.

Hanspeter Kuhn/FMH stellt klar, dass der Fristenlauf nach Empfang der Mitteilung beginnt.

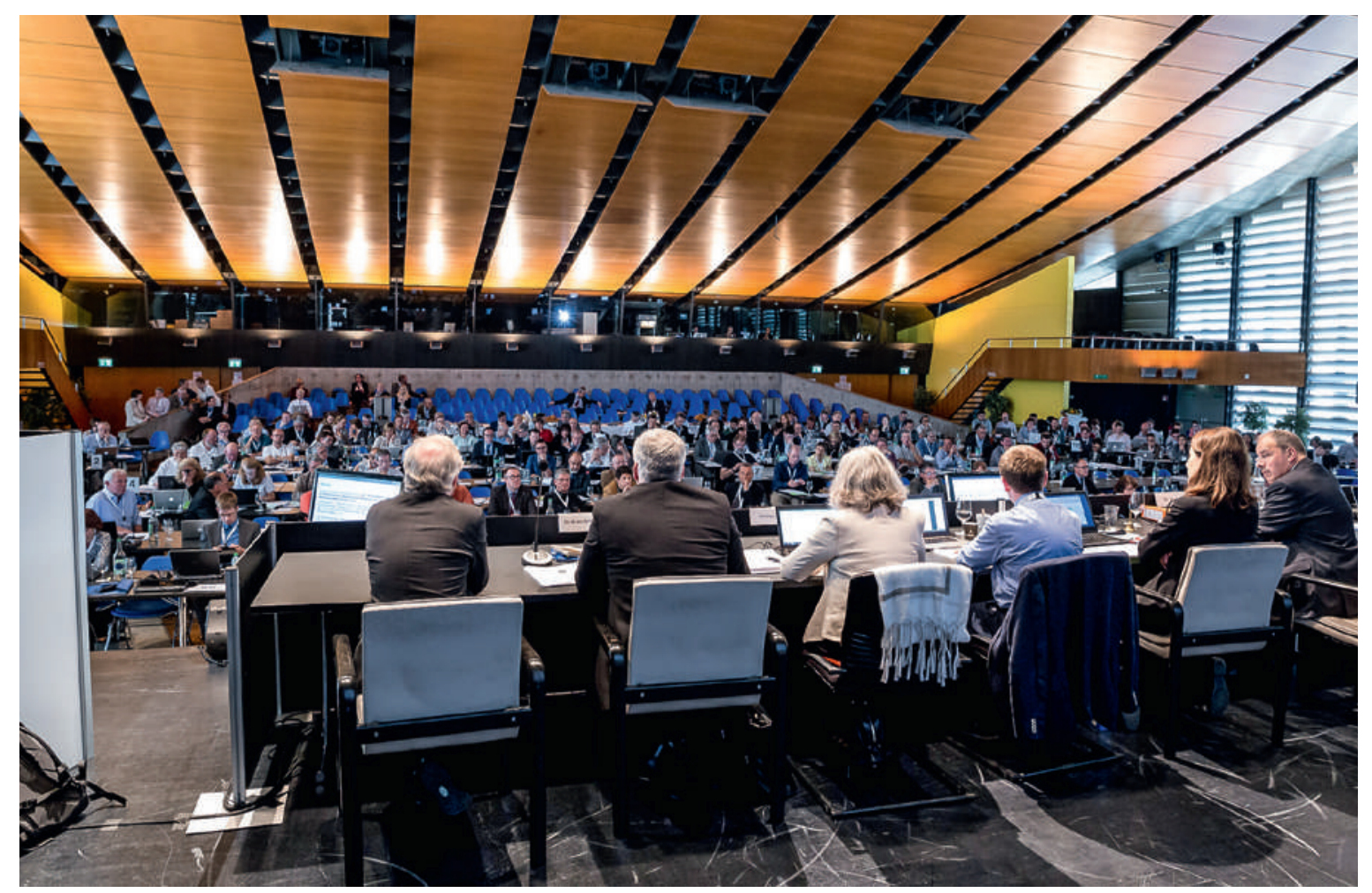

Ein Blick auf das Plenum im Saal aus der Perspektive des FMH-Zentralvorstandes auf dem Podium. 
Antrag:

Ergänzung der GO mit folgender Bestimmung: Art. 3bis Rekursfrist und Rechtsmittelbelehrung bei Ausschluss nach Art. 9 Abs. 4 und 5 der Statuten Die Rekursfrist beträgt 30 Tage seit der Mitteilung. Die Mitteilung erfolgt unter Hinweis auf die Möglichkeit, den Ausschluss innert 30 Tagen an den ZV (beim Ausschluss nach Art. 9 Abs. 4 Statuten) bzw. an die ÄK (beim Ausschluss nach Art. 9 Abs. 5 Statuten) weiterziehen zu können.

Beschluss:

Der Antrag wird mit $162 \mathrm{Ja}$, ohne Gegenstimmen und ohne Enthaltung gutgeheissen.

\section{3 Änderung der FMH-Standesordnung}

\subsection{1 Änderung Art. 44}

$\mathrm{Zu}$ diesem Traktandum werden RA Anne-Sylvie Thiébaud/Rechtsdienst FMH und RA Michel Meier/AeG SO begrüsst. Anne-Sylvie Thiébaud/FMH hält einleitend fest, dass nach den Treffen mit den kantonalen Standeskommissionen im Jahre 2013 die grosse Mehrheit sich für ein einheitliches Verfahrensreglement und die Einrichtung einer Entscheiddatenbank ausgesprochen hat. Der ZV hat daraufhin Anfang 2014 eine Arbeitsgruppe mit der Revision des Reglements der Standeskommission der FMH sowie der Errichtung einer Entscheiddatenbank beauftragt. Die Arbeitsgruppe, unter der Leitung von Jürg Schlup/Präsident FMH, mit den Mitgliedern Dr. René Raggenbass/Präsident der Standeskommission VS, Dr. Marianne Winterhalter/ehemalige Vizepräsidentin der Standeskommission BS, Dr. Urs Strebel/ehemaliger Vizepräsident der Standeskommission FMH, Michel Meier/Rechtsberater AeG Solothurn und Anne-Sylvie Thiébaud/FMH, hat diese Aufgabe an die Hand genommen und dem ZV beantragt, Art. 44 der Standesordnung abzuändern.

Michel Meier führt aus, dass bei Fehlen einer ausdrücklichen Regelung im Verfahrensreglement subsidiär die Bestimmungen der Schweizerischen Zivilprozessordnung (ZPO) gelten soll, anstelle der Bestimmungen des Bundesgesetzes über das Verwaltungsverfahren (VwVG). Das Standesverfahren ist eine Mischung aus Elementen des Straf-, Verwaltungs- und Zivilrechts. Der gegenwärtige Verweis auf das VwVG hat vor allem historische Gründe. Das Zivilverfahren wurde 2011 in der Schweiz einheitlich geregelt und kommt dem Verfahren am nächsten, das vor den Standeskommissionen zur Anwendung gelangt. Für die Basisorganisationen wird diese Änderung im Art. 44 künftig keinen Regelungsbedarf mehr zur Folge haben, soweit das durch die Standesordnung der FMH direkt umgesetzt wird.
Der ZV, die Arbeitsgruppe wie auch die meisten Basisorganisationen, denen der Entwurf des Verfahrensreglements unterbreitet wurde, erachten den Verweis auf die ZPO als sinnvoll. Die Bestimmungen der ZPO gelangen nur zur Anwendung, wenn in einer bestimmten Frage weder der Standesordnung noch dem Verfahrensreglement eine Antwort entnommen werden kann. Der ZV schlägt der ÄK eine Änderung von Art. 44 der Standesordnung der FMH vor, wonach für den Fall des Vorliegens einer Regelungslücke die subsidiäre Anwendbarkeit der ZPO und nicht wie bisher des VwVG vorgesehen wird.

Die DV hat diesen Vorschlag einstimmig unterstützt. Diese Änderung würde bei Annahme nach Ablauf der Einsprachefrist dieses Protokolls in Kraft treten. Die Aufschaltung einer Datenbank ist per 1.1.2016 vorgesehen.

Jürg Schlup/Präsident FMH dankt allen kantonalen Ärztegesellschaften, Anne-Sylvie Thiébaud wie auch Michel Meier für die grosse Arbeit.

\section{Antrag:}

Die ÄK genehmigt folgende Anpassung der Standesordnung der FMH:

Art. 44 (Anwendbares Verfahrensrecht)

Kann in einer bestimmten Frage weder der Standesordnung noch den Reglementen der kantonalen Ärztegesellschaften, des VSAO, des VLSS bzw. für die Standeskommission der FMH eine Antwort entnommen werden, gelten die Bestimmungen der Zivilprozessordnung (ZPO).

Beschluss:

Der Antrag wird mit 149 Ja ohne Gegenstimmen und ohne Enthaltung gutgeheissen.

\subsubsection{Ergänzung Art. 18: Aufnahme Richtlinie der} SAMW betr. Abgrenzung von Standardtherapie und experimenteller Therapie im Einzelfall Die SAMW hat im Mai 2014 die neuen medizin-ethischen Richtlinien und Empfehlungen "Abgrenzung von Standardtherapie und experimenteller Therapie im Einzelfall» beschlossen. Die ÄK hat am 30.10.2014 die Aufnahme dieser Richtlinien in die FMH-Standesordnung aufgrund zweier Interventionen zurückgewiesen. Im Anschluss fanden unter Einbezug der Rückweisenden Konsensgespräche zwischen FMH und SAMW statt. Die bestrittenen Passagen der Richtlinie wurden revidiert. Bei der angebrachten Kritik ging es nicht um die Richtlinie an sich, sondern um die Empfehlungen.

Prof. Christian Kind/Präsident Ethikkommission SAMW stellt die revidierte Richtlinie vor. Ziel ist die Wahrung der therapeutischen Freiheit in schwierigen Einzelfällen, die Verhinderung von Missbrauch wie die 
Festlegung der Abgrenzung zwischen Standardtherapie und experimenteller Therapie. Sie schliesst die vom Gesetzgeber bewusst offengelassene Lücke durch Selbstregulierung der medizinischen Institutionen, wie dies schon die Antwort des Bundesrats vom 23.2.2011 auf die Motion 11.3001 «Heilversuche» als mögliche Lösung erwähnt hatte. Die aufgekommenen Missverständnisse bezogen sich vor allem auf die Frage, was Standardtherapie sei. Standards können auf vielen Ebenen mit unterschiedlichen Zielen definiert werden. Die SAMW-Richtlinie bezieht sich ausschliesslich auf die Ebene der fachlichen Empfehlungen und zielt auf die ärztlichen Sorgfaltspflichten im Einzelfall. Die direkte Anwendung der Definition auf andere Ebenen mit anderen Zielen ist nicht intendiert.

Bei den Konsensgesprächen hat man sich darauf geeinigt, den Einbezug von Vertrauenspersonen bei der Aufklärung über experimentelle Therapien verbindlich zu erklären. Die Patienten können eine Vertrauensperson mitbringen. Die Tabelle über die verschiedenen Ebenen der Standardisierung wurde aus der Richtlinie entfernt. Die SAMW hat aufgrund dieser Missverständnisse entschieden, künftig ihre Empfehlungen zu Richtlinien separat zu publizieren.

Der ZV beantragt der ÄK, die Richtlinie in der neuen Fassung in die FMH-Standesordnung aufzunehmen. Die Übernahme der Richtlinie in die FMH-Standesordnung soll Arzt und Ärztin eine klare Handlungsanweisung und den Patienten Sicherheit geben. Der Beschluss der ÄK erfolgt unter Vorbehalt der Zustimmung des Senats der SAMW zur Richtlinienrevision. Dieser tagt am 19.5.2015.

Charles-A. Favrod-Coune/VD, Mitglied Standeskommission FMH weist auf die Wichtigkeit der Richtlinie in der Standesordnung hin. Er stellte heute morgen fest, dass die SAMW nach wie vor die alte Version publiziert hat. Seiner Meinung nach können nicht zwei unterschiedliche Versionen bestehen. Es muss sichergestellt sein, dass die SAMW die Richtlinie ohne Empfehlungen publiziert und die Richtlinie keinen Bezug auf die Empfehlungen nimmt. Weiter moniert er, dass sowohl die SAMW wie auch die FMH diese Richtlinie zur gleichen Zeit publizieren. Seiner Meinung nach ist das Kompendium nicht mehr komplett und die französische Version der Übersetzung ist weder stimmig noch entspricht sie den Gender-Anforderungen.

Was die gemeinsame Publikation betrifft, weist Hanspeter Kuhn/FMH darauf hin, dass die SAMW nach dem Entscheid des Senats, welcher am 19.5.2015 tagt, die Richtlinie sofort in Kraft setzen kann. Bei der FMH ist jedoch die 60-tägige Einsprachefrist nach Publikation des Protokolls abzuwarten.
Prof. Christian Kind/SAMW kennt die Gender-Problematik, will jedoch an der vorliegenden französischen Version festhalten. Die SAMW verwendet in der Regel nur eine Form und verweist in einer Fussnote, dass das andere Geschlecht auch gemeint ist. Was das Kompendium betrifft, bittet er Charles-A. Favrod-Coune, mit ihm dies bilateral zu klären.

Die beiden Einsprechenden aus der ÄK vom 30.10.2014, Jürg Nadig und Beat Gafner, sind mit der überarbeiteten Version einverstanden und danken, dass auf ihre Kritikpunkte eingegangen wurde. Jürg Nadig hält fest, dass seine Fachgesellschaft hier besonders gefordert sei. Vorgesehen ist ein Beitrag in der $S \ddot{A} Z$, in welchem definiert ist, was aus Sicht der Fachgesellschaft als Standardtherapie gelten soll, damit die Abgrenzung zur experimentellen Therapie gemacht werden kann.

Antrag:

Ergänzung FMH-Standesordnung: Art. 18 Richtlinien der Schweizerischen Akademie der Medizinischen Wissenschaften (Ergänzung)

Bezüglich [...] Abgrenzung von Standardtherapie und experimenteller Therapie im Einzelfall gelten die Richtlinien der Schweizer Akademie der Medizinischen Wissenschaften. [...]

\section{Beschluss:}

Dem Antrag wird mit 158 Ja und 1 Enthaltung zugestimmt.

Der Beschluss der ÄK erfolgt unter Vorbehalt der Zustimmung des Senats der SAMW zur Richtlinienrevision. Dieser tagt am 19.5.2015.

\subsubsection{Aktualisierung Art. 18: Aufnahme der} revidierten Richtlinien "Ausübung der ärztlichen Tätigkeiten bei inhaftierten Personen" Gemäss Monique Gauthey/ZV FMH war und ist die Mitwirkung bei Ärzten, insbesondere bei der Ausschaffung von aus der Schweiz ausgewiesenen Personen, heikel und nicht praxisnah geregelt. Dies bewegte die Konferenz der Kantonalen Justiz- und Polizeidirektorinnen und -direktoren (KKJPD), Bundesrätin Sommaruga am 15.5.2013 den Vorschlag zu unterbreiten, dass Bund und Kantone unter Einbezug der Nationalen Kommission zur Verhütung von Folter (NKVF) mit der Ärzteschaft in einen Dialog eintreten sollen. Ziele dieses Dialogs waren insbesondere die medizinische Begleitung von Rückführungen transparenter zu machen im Hinblick auf eine breite Abstützung unter der Ärzteschaft; das Vertrauen der Ärzteschaft in die medizinische Begleitung zu erhöhen; die Problematik des medizinischen Datenflusses im Vorfeld von Rückführungen zu thematisieren. Nach Analyse der komplexen Situation und Klärung der umstrittenen Punkte und der verschiedenen Rollen hat die SAMW in Ergän- 


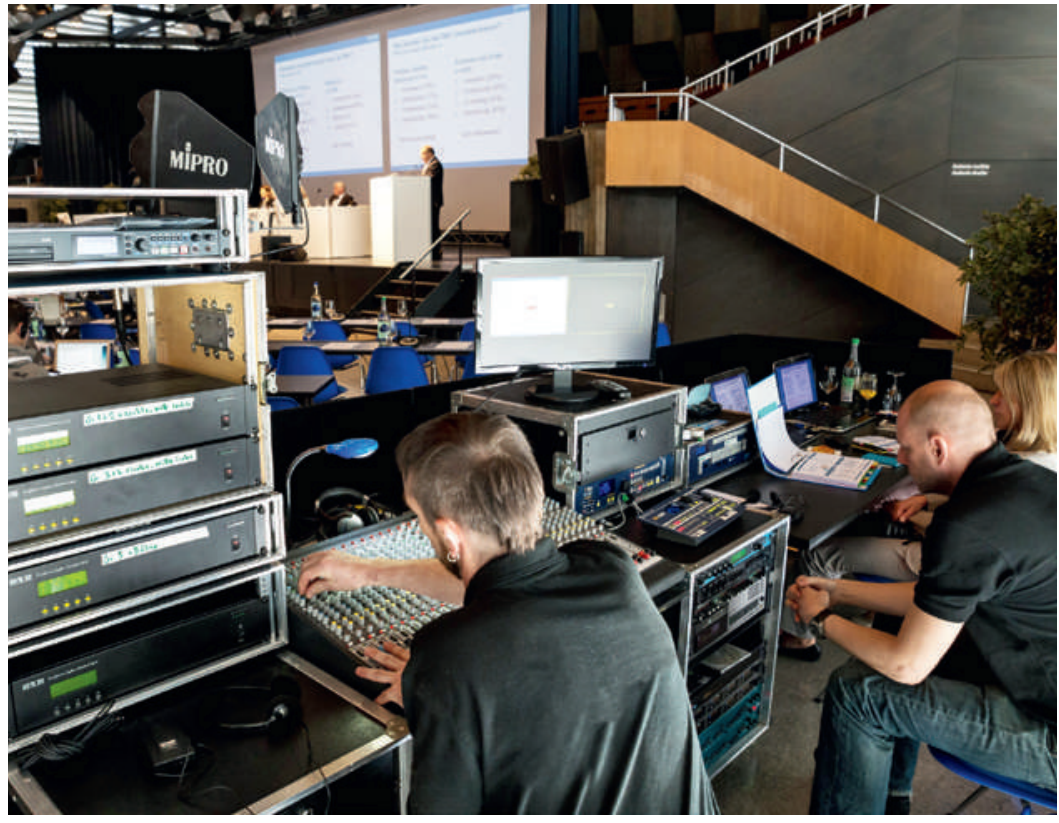

Die Saaltechniker tragen hinter den Kulissen zu einer gelungenen Versammlung bei.

zung zu den seit 2013 stattfindenden Diskussionen zwischen Staatssekretariat für Migration, SAMW und FMH zur Rolle der Ärztinnen und Ärzte im Zusammenhang mit zwangsweisen Rückführungen von abgewiesenen Asylsuchenden die Richtlinien «Ausübung der ärztlichen Tätigkeit bei inhaftierten Personen» revidiert. Diese Richtlinien beschreiben die Aufgaben der Ärzteschaft, wie sie sich aus dieser neuen Rollenteilung ergeben. Die neue Rolle soll in den kommenden Monaten evaluiert werden, um festzustellen, ob damit die medizinischen Risiken vermindert werden können.

Prof. Christian Kind/SAMW informiert über die neue Regelung, die für alle Ärzte Verbindlichkeit hat. Ein neuer Anhang zur bestehenden Richtlinie sowie die Publikation einer Kontraindikationen-Liste für den Ausschaffungsflug sollen Klarheit bringen. Die Flugfähigkeit, das "fit to fly", wird in Zukunft nicht mehr durch den behandelnden Arzt, sondern durch den Begleitarzt beurteilt. Dieser entscheidet letztlich über die Flugfähigkeit. Das Staatssekretariat für Migration hat mit der Firma Oseara AG, die solche Flüge professionell begleitet, eine entsprechende Vereinbarung abgeschlossen. Thomas Heuberger/BE will wissen, inwieweit die Frage der zwangsweisen Behandlung während eines Flugs geklärt ist und ob im Falle eines Falles die Entscheidungsfähigkeit beim Flugkapitän liegt.

Prof. Christian Kind/SAMW weist darauf hin, dass in den Richtlinien die Frage der zwangsweisen Behandlung erwähnt ist. Eine Zwangsbehandlung ist nur unter den gleichen Bedingungen möglich wie bei nichtinhaftierten Personen. Die Urteilsunfähigkeit wie auch die Situation, dass keine andere Möglichkeit da ist, dem Patienten zu helfen, muss gegeben sein. Die Richtlinien für die Zwangsbehandlung werden zurzeit ausgearbeitet und den Delegierten voraussichtlich in der nächsten ÄK vorgelegt. Seiner Meinung nach kann der Flugkapitän sagen, was mit dem Passagier geschieht, nicht aber, was medizinisch gemacht werden muss. Der Begleitarzt entscheidet, ob die Behandlung durchgeführt wird oder nicht.

Thomas Heuberger/BE befremdet diese Haltung, weil die Verantwortung für den Flug und die Passagiere letztendlich beim Flugkapitän liegt. Wenn es zu Zwischenfällen kommen sollte, die die Flugsicherheit gefährden, sei die Meinung des Flugkapitäns sehr wohl wichtig. Prof. Christian Kind/SAMW meint, dass der Flugkapitän vom Arzt verlangen kann, dass der Patient die Flugsicherheit nicht gefährdet. Wie der Arzt das jedoch macht, liegt in seiner Verantwortung.

Daniel Jud/TG wünscht weitere Informationen über die Firma Oseara.

Prof. Christian Kind/SAMW verfügt über keine weiteren Informationen und verweist auf die Website. Die Firma wurde eigens für diesen Zweck gegründet. Der leitende Arzt, Dr. Adrian Businger, war ebenfalls an den Gesprächen anwesend.

Prof. Jean-Michel Gaspoz/SGIM dankt als Departementschef, Département de médecine communautaire, de premier recours et des urgences HUG, dem auch die Gefängnismedizin unterstellt ist, für die vorliegende Richtlinie, die die Rollen der Beteiligten klärt. Wichtig ist für ihn, dass der behandelnde Arzt seine Rolle kennt und wahrnimmt und dass dieser nicht ein Zertifikat «fit to fly" ausstellen muss und so durch die politischen und polizeilichen Autoritäten instrumentalisiert wird. Die Rolle des behandelnden Arztes ist, die Informationen des Gesundheitszustands des Patienten festzuhalten, und, vorausgesetzt, dieser ist einverstanden, zuhanden des für die Beurteilung der Flugfähigkeit zuständigen Arztes mitzuteilen. Er trägt jedoch nicht die Verantwortung für das, was durch eine administrative oder polizeiliche Verfügung ausgelöst werden könnte.

\section{Antrag:}

Der ZV beantragt der ÄK die Aktualisierung des Verweises auf die SAMW-Richtlinien "Ausübung der ärztlichen Tätigkeit bei inhaftierten Personen":

Bezugnahme auf die revidierte Richtlinie.

Beschluss:

Dem Antrag wird mit 152 Ja und 1 Gegenstimme zugestimmt. Der Beschluss der ÄK erfolgt unter Vorbehalt der Zustimmung des Senats der SAMW zur Richtlinienrevision. Dieser tagt am 19.5.2015. 


\section{Wahlen, Bestätigungswahlen, Ersatzwahlen}

\subsection{Bestätigungswahl von nominierten DV-Delegierten}

\subsection{Bestätigungswahl von nominierten Ersatz- delegierten}

Zu 6.1 und 6.2 wurden wohl Ankündigungen gemacht, aber schlussendlich keine Meldungen eingereicht. Aus diesem Grunde erübrigen sich Bestätigungswahlen. Jürg Schlup/Präsident FMH nimmt die Gelegenheit wahr, die Delegierten über die Gründe zu informieren, warum der ZV keine Ersatzwahl für den verstorbenen Vizepräsidenten Ernst Gähler beantragt hat. Einerseits lief die ordentliche Frist zur Einreichung einer Kandidatur zwei Tage nach den Begräbnisfeierlichkeiten von Ernst ab. Anderseits schlägt die Taskforce der ÄK heute eine Verkleinerung des ZV vor. Der ZV wird den heutigen Entscheid der ÄK abwarten und dann eine Ersatzwahl für die kommende ÄK planen.

\section{Finanzplanung/Finanzkontrolle}

\subsection{Leistungsverträge der FMH}

Anne-Geneviève Bütikofer/GS FMH erläutert die Gründe, die den ZV 2013 im Rahmen der finanziellen Stabilisierung bewogen haben, mit allen Leistungsempfängern, die von der FMH jährlich mit mehr als CHF 10000 unterstützt werden, einen Leistungsvertrag abzuschliessen. Ein entsprechendes Ablaufverfahren wurde den Delegierten an der ÄK vom 3.10.2013 vorgestellt. Einige Partner stellten jedoch die Entscheidungsgrundlage, die diesen Leistungsverträgen zugrunde liegt, in Frage. Am 8.5.2014 nahm die ÄK Kenntnis von den neuen Leistungsverträgen und dem finanziellen Engagement zwischen FMH und Leistungsempfänger. Die meisten Partner haben sich mit dem unterbreiteten Entwurf des Leistungsvertrages einverstanden erklärt, einige stützten sich jedoch auf einen früheren ÄK-Beschluss und machten geltend, dass dieser eine ausreichende rechtliche Grundlage sei. Eine formelle Bestätigung war für die ÄK vom 30.10.2014 vorgesehen. Die Einholung des formellen Beschlusses musste jedoch aufgrund der fehlenden Zeit auf heute verschoben werden. Fiorenzo Caranzano/TI hat im Prinzip nichts gegen den Abschluss dieser Leistungsverträge. Er wünscht jedoch, dass die Verträge den effektiven Tatsachen angepasst werden.

Anne-Geneviève Bütikofer/GS FMH dankt für die Präzisierung und informiert, dass das Generalsekretariat
FMH in der Zwischenzeit von den betroffenen Organisationen die Zusicherung für den Abschluss der geforderten Leistungsverträge erhalten hat. In einer konstruktiven Diskussion konnten die teilweise komplexen und völlig verschiedenen Fragestellungen vor allem bei den Zeitschriftenverlagen geklärt werden. Der Leistungsvertrag soll das Verhältnis und die Modalitäten zwischen dem Erbringer und dem Käufer der Leistung regeln und wird von der Abteilung Verwaltung und Finanzen kontrolliert. Das Verfahren entspricht dem bereits früher präsentierten Ablauf.

\section{Antrag:}

Die ÄK beschliesst, dass die FMH mit allen Partnern, welche von der FMH eine finanzielle Unterstützung von mehr als CHF 10000 pro Jahr erhalten, einen Leistungsvertrag abschliessen muss. Dieser Leistungsvertrag wird zwischen der FMH und dem Leistungsempfänger abgeschlossen bzw. erneuert, bevor eine Zahlung fliesst.

\section{Beschluss:}

Dem Antrag wird mit 152 Ja und 4 Enthaltungen zugestimmt.

\section{Budgetstabilisierung der FMH}

Jürg Schlup/Präsident FMH informiert, dass das Eintreten auf dieses Geschäft mit den Anträgen des VSAO auf Nichteintreten und des SMSR auf Rückweisung bestritten wird. Aufgrund dieser Anträge muss zuerst eine Eintretensdebatte geführt werden, dann über diese beiden Anträge abgestimmt werden. Anschliessend folgt die Detailberatung. Zu diesem Ablauf hat unser Rechtsdienst ein Gutachten bei Prof. H. M. Riemers erstellen lassen. Die Eintretensdebatte wird wie folgt ablaufen: Zuerst das Einleitungsvotum der Taskforce, dann das Ergänzungsvotum der GPK, dann Antragsteller VSAO, Antragsteller SMSR, ZV FMH, Rechtsdienst FMH, Eintrittsdiskussion Plenum und schliesslich das Schlussvotum der Taskforce. Anschliessend wird über Nichteintreten oder Rückweisung abgestimmt.

\subsection{Eintretensdebatte}

Josef Widler/AGZ stellt den Antrag, die Redezeit der Eintretensdebatte auf zwei Minuten zu beschränken.

Ordnungsantrag Josef Widler:

Die Redezeit für die Eintretensdebatte ist auf zwei Minuten zu beschränken.

\section{Beschluss:}

Dem Antrag wird mit 120 Ja, 10 Nein und 6 Enthaltungen zugestimmt.

Felix Kurth/VSAO ist der Meinung, dass es sich hier um zwei verschiedene Anträge handelt. Der VSAO verlangt Nichteintreten und keine Präsentation, die SMSR will 
die Taskforce anhören und erst anschliessend den Bericht zurückweisen. Man kann später nicht noch einmal abstimmen, ob die Präsentation gezeigt werden soll oder nicht.

Daniel Schröpfer/VSAO stellt klar, dass im Antrag des SMSR die Präsentation vorgesehen ist. Insofern stellt sich die Frage, warum die Abstimmung über Präsentation oder nicht jetzt schon durchgeführt werden soll. Hanspeter Kuhn/FMH empfiehlt, wie vorgesehen, in der Eintretensdebatte kurze Voten abzugeben, aber nicht die ganze Präsentation anzuhören. Das ist auch die Empfehlung von Prof. Riemer. Das Gutachten von Prof. H.M. Riemer kommt zum Schluss, dass die Traktanden gehörig anzukünden sind. Dazu braucht es Unterlagen mit allen Informationen, um sich für die Entscheidfindung gut vorzubereiten. Gemäss Prof. H.M. Riemer war dies im vorliegenden Fall nicht so und diese Pflicht wurde in zwei Punkten verletzt. Die Taskforce hat nur ein kurzes Papier und nicht die gesamte Analyse den Delegierten zur Verfügung gestellt. Und die Delegierten erhielten auch nicht das Protokoll der Diskussion in der DV zu diesem Thema.

Beat Gafner/BE beantragt, die Präsentation der KPMG vorzuziehen.

\section{Antrag Gafner:}

Die Präsentation der KPMG ist vorzuziehen. Nur unter Kenntnis dieser Präsentation kann über das weitere Vorgehen beraten und entschieden werden.

Beschluss:

Der Antrag wird mit 41 Ja, 91 Nein und 11 Enthaltungen abgewiesen.

Nach der Ablehnung des Antrags Gafner stellt die BEKAG einen weiteren Antrag. Sie wollen die Präsentation der KPMG auch dann sehen, wenn die Detailberatung nicht zustande kommt.

Antrag BEKAG:

Falls die Detailberatung nicht zustande kommt, soll die Präsentation der KPMG gleichwohl gezeigt werden.

Beschluss:

Der Antrag wird mit 39 Ja, 83 Nein und 7 Enthaltungen abgewiesen.

\subsubsection{Einleitungsvotum Taskforce}

Peter Wiedersheim/Taskforce zitiert den Protokollauszug der ÄK vom 30.10.2014. Diesem kann entnommen werden, dass 95\% der Einnahmen für gebundene Ausgaben reserviert sind. Dadurch ergibt sich kein Handlungsbedarf für neue dringliche Aufgaben und es besteht eine Diskrepanz zwischen Einnahmen und Ausgaben. Die ÄK hat am 30.10.2014 eine Taskforce mit folgenden Zielvorgaben eingesetzt: Ausgaben und Ein- nahmen sind dauerhaft im Gleichgewicht; Schaffung eines operativen Freiraums von mindestens 20\% für priorisierte Projekte; Abbau von Nebenaufgaben; Erarbeitung von neuen Finanzierungsmöglichkeiten.

Die DV hat an ihrer Sitzung vom 26.11.2014 zuerst den Protokollführer, Daniel Trachsel, und mit klarem Mehr folgende Mitglieder gewählt: Adrian Sury/GPK, Alexander Zimmer/GPK, Brigitte Muff/DV, Pierre Vallon/ DV, Peter Wiedersheim/DV. Da Brigitte Muff diese Wahl abgelehnt hat, wurde an ihre Stelle Karin Stadlin gewählt. Die Leitung der Taskforce wurde dem externen Ökonom, Dr. Walter Gratzer/KPMG, übertragen. Vom GS FMH wurde Emanuel Waeber/Leiter Verwaltung und Finanzen als Gast eingeladen.

Peter Wiedersheim erinnert die Delegierten, dass die ÄK die Auftraggeberin war. Die Taskforce als Auftragnehmerin ist somit der ÄK gegenüber rechenschaftsund informationspflichtig. Dieser Pflicht wird mit der heutigen Information nachgekommen. Für die Schaffung eines operativen Freiraums von 20\%, was ungefähr CHF 4-6 Mio. entspricht, war für die Taskforce klar, dass diese Vorgabe primär nur mit einer Kostenreduktion und sekundär mit der Erhöhung der Mitgliederbeiträge erreicht werden kann. Er stellt klar, dass die Taskforce kein Grounding der FMH will, sondern eine starke FMH mit einer optimalen Flugtauglichkeit. Die aktuellen Finanzen seien jedoch kein Zeichen von Stärke. Die Führung wird immer mehr zu einer überwiegenden Verwaltung reduziert. Für die Führung der Organisation braucht der Präsident genügend Ressourcen. Das Aufgabenpensum der FMH ist gross und wird sich weiter erhöhen. Der Antrag des VSAO löst diese Probleme nicht, sondern schwächt den Verband. Die Taskforce empfiehlt, den Antrag VSAO abzulehnen. Nur so kann sie ihrem Informationsauftrag an der heutigen ÄK nachkommen. Er bittet, zumindest die Ergebnisse präsentieren zu können.

\subsubsection{Ergänzungsvotum GPK}

Adrian Sury/GPK verzichtet auf sein Votum, da er mit Peter Wiedersheim das Einleitungsvotum der Taskforce besprochen hat.

8.1.3 Antrag VSAO: Nichteintreten auf die Budgetstabilisierungsanträge der Taskforce und neuer Auftrag an den ZV der FMH

Daniel Schröpfer/VSAO bedankt sich bei der KPMG und der Taskforce für die wertvolle Arbeit. Er unterstreicht, dass der VSAO schon seit Jahren auf Sparpotentiale bei der FMH hinweist und angeregt hat, die Prozesse kritisch zu hinterfragen und Überlegungen anzustellen, wofür die FMH das Geld ausgibt. Der VSAO unterstützt seit 2007 die Sparbemühungen der FMH und will mit 
seinem Antrag keineswegs die Arbeit schlecht machen, oder wie Herr Wiedersheim vorhin meinte, die FMH ins Wanken bringen. Vielmehr bringt der Antrag der Taskforce für die FMH eine Destabilisierung. Mit der vorgeschlagenen Weise kann nicht gespart werden. Auch ist der VSAO mit dem gewählten Vorgehen nach Abschluss der Arbeiten nicht einverstanden. Weder der ZV noch das Generalsekretariat wurden bei der Umsetzung der Massnahmen miteinbezogen. Heute soll über ein Sparpaket abgestimmt werden, ohne zu wissen, was denn konkret gespart werden soll. Der VSAO fordert deshalb, dass der Bericht der Taskforce in seiner Gesamtheit den Delegierten der ÄK rechtzeitig vorgelegt wird und die wichtigen Akteure in die Umsetzung einbezogen werden. Basierend auf einer neuen Auslegeordnung kann die ÄK im Oktober 2015 über konkrete Massnahmen entscheiden.

\subsubsection{Antrag SMSR: Rückweisung des Berichts der Taskforce Budgetstabilisierung an den $Z V$} Pierre-Alain Schneider/GE hat die Auslotung der Sparmöglichkeiten durch die Taskforce geschätzt und ist der Meinung, dass die ÄK diesen Bericht zur Kenntnis nehmen soll. Wie bereits andere Delegierte sich äusserten, war die SMSR über die Art der Anträge und der geplanten Vorgehensweise, ohne vorherige Analyse mit dem ZV und dem GS FMH, geschockt. Die Taskforce hat ihre Aufgabe gemacht, aber aufgrund der vorgelegten Unterlagen kann die ÄK keinen Entscheid zu den Sparmassnahmen fällen. Der Bericht muss zuerst im ZV beraten und dann mit dem Generalsekretariat FMH besprochen werden. Wir vertrauen diesen beiden Gremien. Der Bericht der Taskforce schafft jedoch Ver-

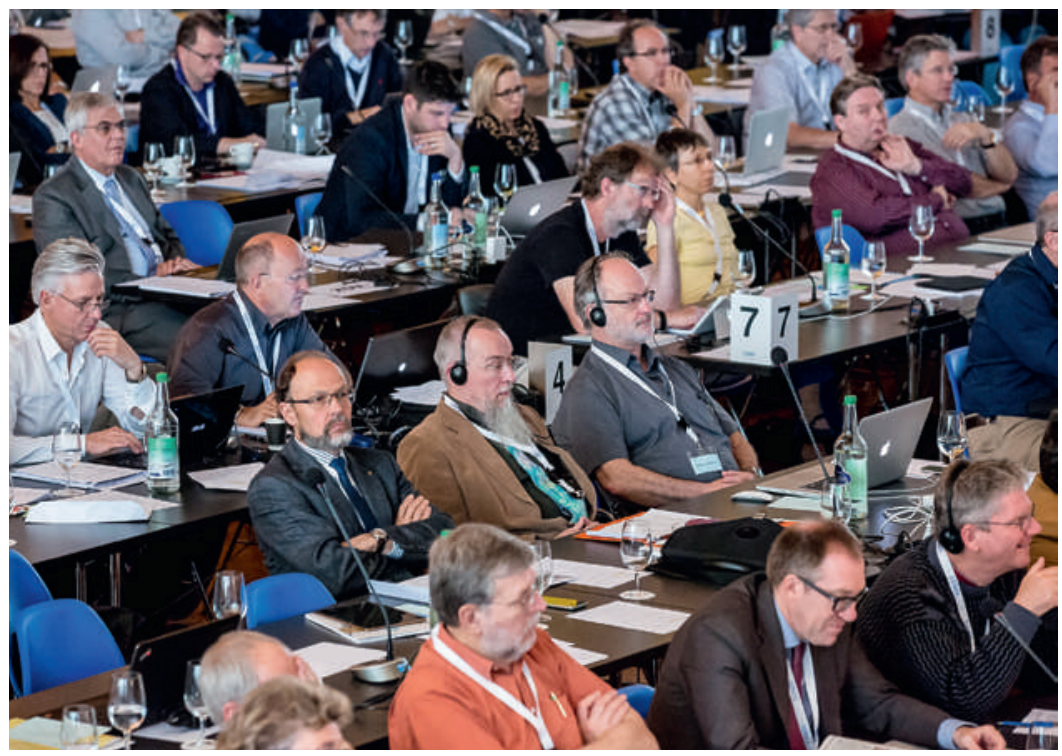

Die Ärztekammer-Delegierten verfolgen gespannt die Debatten, um anschliessend ihre Beschlüsse zu treffen. unsicherung und erweckt den Eindruck, dass der Verband am Untergehen ist. Aus diesem Grunde schlägt er vor, dass der ZV die Massnahmen nun analysiert und vertieft.

\subsubsection{Empfehlungen ZV}

Jürg Schlup/Präsident FMH dankt der KPMG und der Taskforce für die Übernahme der schwierigen und anspruchsvollen Aufgabe einer Analyse der FMH und der Ausarbeitung eines entsprechenden Berichts. Der ZV ist sich bewusst, dass aufgrund der Vorgaben der ÄK für die Analyse und der Erstellung des Berichts sehr wenig Zeit zur Verfügung stand.

Der ZV stabilisiert seit Anfang 2013 - seit gut zwei Jahren - das FMH-Budget. Er hat dazu die folgenden sechs Massnahmen konkret realisiert, je drei zur Ertragsverbesserung und drei zur Ausgabenreduktion. Der ZV hat zur Ertragsverbesserung die Mitgliederbeiträge der Kategorie 3 erhöht, die Kategorie 10 neu geschaffen sowie die Kategorien 7 und 9 neu definiert. Weiter hat der ZV zur Ausgabenreduktion eine Ausgabenbremse beschlossen, neue Kommissionen einer Bewilligungspflicht unterstellt und ein neues Sitzungsentschädigungsreglement eingeführt, welches ebenfalls bisherige Ausgaben reduziert. Zusätzlich hat der ZV der DV im September 2014 eine Liste der Aufgaben der FMH vorgelegt und die DV gebeten, diese Aufgabenliste zu überprüfen mit der Frage: Was soll die FMH weiterhin tun und was nicht mehr? Der ZV bemüht sich seit 2013, also seit zwei Jahren, um eine schrittweise Stabilisierung des Budgets mittels Ertragsverbesserung und Ausgabenreduktion. Der ZV hat auch den Antrag der GPK an der ÄK vom 30.10.2014 unterstützt, weil der ZV den FMH-Haushalt im Lot haben will.

Vier Wochen nach der letzten ÄK, am 26.11.2014, legte die DV die personelle Zusammensetzung der Taskforce Budgetstabilisierung fest und schloss dabei sowohl den ZV als auch die GS aus der Taskforce aus. Dieser Ausschluss ist aus Sicht des ZV unverständlich und nicht zielführend, eine solche strategische Aufgabenüberprüfung ohne das strategische Führungsorgan der FMH wahrzunehmen. Zudem führt das vorgelegte fünfseitige Dokument mit den vorgeschlagenen Massnahmen im Generalsekretariat zu Verunsicherung und Demotivation. Der ZV will den FMH-Haushalt im Lot haben. Und die Delegierten wollen von der FMH gute Dienstleistungen von motivierten Leuten. Nun gilt es, behutsam und intelligent vorzugehen. Ein Stellenabbau oder eine Stellenverschiebung löst innerhalb des Generalsekretariats massive Unruhe und Reibungsverluste aus. Unreflektierte Massnahmen können die FMH nachhaltig schädigen. Er unterstreicht nochmals, 
dass der ZV sich seit zwei Jahren mittels Ertragsverbesserung und Ausgabenreduktion um ein ausgeglichenes Budget bemüht und der ÄK im kommenden Herbst ein ausgeglichenes Budget unterbreiten wird. Der ZV wird nach Prüfung der Berichtsergebnisse der Taskforce der ÄK am 29.10.2015 eine Liste mit umsetzbaren Vorschlägen präsentieren, aufgrund deren die Delegierten klare Entscheide fällen können.

\subsubsection{Rechtsdienst FMH}

Hanspeter Kuhn/FMH erwähnt, dass die Frage für die richtige Vorgehensweise wichtig schien, weshalb Prof. H. M. Riemer kontaktiert wurde. Vereinsrechtlich stellte sich weiter die Frage, ob die von der Taskforce an die Delegierten zugestellten Unterlagen für eine Entscheidfindung ausreichend waren. Ein Gutachten, welches bei Prof. H.M. Riemer in Auftrag gegeben wurde, kommt zum Schluss, dass aufgrund von Art. 67 Abs. 3 ZGB jene Traktanden, über die Beschluss gefasst werden soll, gegenüber den Stimmenden gehörig anzukündigen sind, damit diese sich entsprechend vorbereiten können. Dazu gehört auch die Zusendung oder wenigstens die Mitteilung der Abrufbarkeit (Internet, Intranet) der einschlägigen Unterlagen, da deren Studium für eine sachgerechte Vorbereitung unerlässlich ist. Das Gutachten zeigt, dass im vorliegenden Geschäft die Unterlagen, die den Delegierten von der Taskforce zugestellt wurden, nicht für eine sachgerechte Entscheidung ausreichen. Die Pflicht zur gehörigen Ankündigung wurde zweimal verletzt. Es wurde der Einladung vom 7.5.2015 lediglich eine kurze Zusammenfassung von fünf Seiten beigelegt. Die ÄK-Delegierten erhielten, entgegen Art. 20 Abs. 3 GO, auch den einschlägigen Protokollauszug (Ziffer 13) der DV vom 18.3.2015 nicht zugestellt, welcher wohl zusätzliche Entscheidelemente für die heutige Sitzung geliefert hätte.

Wie eingangs schon erwähnt, kommt das Gutachten von Prof. H.M. Riemer zum Schluss, dass die Traktanden gehörig anzukünden sind. Dazu braucht es Unterlagen mit allen Informationen, um sich für die Entscheidfindung gut vorzubereiten. Im vorliegenden Fall wurde jedoch diese Pflicht - wie bereits oben erwähnt -in zwei Punkten verletzt.

\subsubsection{Eintrittsdiskussion Plenum}

Es gibt folgende Wortmeldungen:

Josef Widler/AGZ erinnert daran, dass die ÄK der Taskforce den Auftrag für die Prüfung von möglichen Massnahmen erteilt hat. Diese liegen nun vor. Seiner Meinung nach muss heute nicht beschlossen werden, ob diese Vorschläge umzusetzen sind. Er vertritt auch nicht die Ansicht des ZV, dass es falsch war, dem ZV kei- nen Einsitz in der Taskforce zu gewähren. Er empfiehlt den Delegierten, auf die Detailberatung einzutreten. Die AGZ wird dann einen Gegenantrag stellen, der das weitere Vorgehen zum Beschluss vorlegen wird. Aber natürlich erst, wenn Eintreten beschlossen wurde. Alex von Weymarn/med-swiss.net unterstützt es, dass zumindest die Arbeiten der Taskforce angehört werden. Was dann entschieden werden soll, ist eine andere Frage.

Jean-Pierre Pavillon/VD gibt zu bedenken, dass Entscheidungen, die zu schnell getroffen werden, meist schlechte Entscheidungen sind. Gute Entscheidungen brauchen Zeit. Seit sieben oder acht Jahren wird schon der Teufel an die Wand gemalt, aber heute von katastrophalen FMH-Finanzen zu sprechen, ist grundlos. Er beantragt, dass die Delegierten die Arbeit der Taskforce zur Kenntnis nehmen. Der ZV soll nach Analyse dieser Vorschläge an der nächsten ÄK geeignete und umsetzbare Massnahmen unterbreiten.

Monique Lehky Hagen/VS widerspricht dem Vorredner. Ihrer Meinung nach handelt es sich bei der heute präsentierten Jahresrechnung um eine schön frisierte kosmetisch verhübschte Rechnung. Dieses Resultat ist vor allem auf die Nichtrealisierung von Projekten zurückzuführen. Es gibt keine Reserven und es ist nicht korrekt, von einem ausgeglichenen Budget zu sprechen.

Christian Bernath/FMPP meint, dass die Taskforce ihren Auftrag erfüllt hat. Er findet es jedoch nicht richtig, heute schon Entscheide zu fällen, ohne zu wissen, welche Konsequenzen dies hat. Der Entscheid, Dienstleistungen zu reduzieren, liegt bei der ÄK. Es könnte ja sein, dass die ÄK nicht darauf eingeht und viel eher die Mitgliederbeiträge erhöhen will.

Für Thomas Heuberger/BE gehen die Anträge VSAO und SMSR in eine ähnliche Richtung. Der Unterschied besteht einzig darin, dass der VSAO die Auflösung der Taskforce beantragt. Wichtig erscheint ihm die Gemeinsamkeit in den Anträgen, dass die Exekutive ihre Aufgabe übernehmen kann. In diesem Sinne findet er es richtig, dass man den Vorschlag der Taskforce zurückweist, aber dann in der nächsten ÄK die entsprechenden Vorschläge, die auch tragbar sind und den ZV und das Generalsekretariat FMH nicht blockieren, diskutiert. Er plädiert für die Annahme der beiden Anträge, da diese identisch sind.

Pierre Vallon/Taskforce will vom Rechtsdienst wissen, ob die alleinige Einsicht des vollständigen Berichts die Taskforce gegen eine juristische Auseinandersetzung bzw. gegen die Entscheidung, die heute getroffen werden konnte, schützt.

Hanspeter Kuhn/FMH interpretiert die Haltung von Prof. Riemer derart, dass die Informationen zu diesem Thema 
nicht ausreichend waren, um eine wasserdichte Entscheidungsfindung heute zu treffen. Das Risiko einer Anfechtung ist gegeben, weshalb Prof. Riemer empfiehlt, keine abschliessenden Entscheide heute zu fällen.

Daniel Ackermann/SGU meint, dass es heute nicht darum geht, über Details zu entscheiden, sondern darum, ob man die Informationen der Taskforce anhören will oder nicht. Da unterscheidet sich der Antrag vom VSAO von jenem des SMSR ganz entscheidend. Die Konsequenzen sind anders. Die ÄK hat im letzten Jahr einen Auftrag erteilt und hat nun das Anrecht, alle Informationen zu erhalten. Der ZV wie auch die GS FMH sind in den nächsten Monaten für die Ausarbeitung der Konsequenzen und Umsetzung der Massnahmen verantwortlich.

Rolf Hunkeler/AGZ fragt sich, warum die Taskforce abzuschaffen sei, wenn die Exekutive von den Vorschlägen überzeugt ist. Er schlägt vielmehr eine Zusammenarbeit vor. In diesem Sinne unterstützt er den Antrag des VSAO wegen dem Abschaffen der Taskforce nicht, sondern jenen des SMSR. Er unterstützt im Prinzip diesen Antrag, jedoch ohne Abschaffung der Taskforce.

Jürg Schlup/Präsident FMH entgegnet, dass es sich hier um einen neuen Antrag handeln würde.

Michaël Hagmann/VD unterstützt den Antrag Hunkeler. Wenn heute auf Nichteintreten plädiert wird, soll die Taskforce von der DV aufgefordert werden, mit dem ZV zusammenzuarbeiten, damit ein Kompromiss gefunden werden kann. Die Taskforce soll beibehalten und die gemeinsam erarbeiteten Vorschläge an der ÄK vom 29.10.2015 präsentiert werden.

Anmerkung: Rolf Hunkeler/AGZ hat diesen Antrag am Schluss der Eintrittsdebatte zurückgezogen.

Hans-Anton Vogel/VEDAG hält fest, dass die Taskforce den von der ÄK erteilen Auftrag erfüllt hat. Wenn die Informationen vorhanden sind, können weitere Schritte miteinander diskutiert werden. Daher fordert er zuerst die Informationen und dann ein Weitermachen.

Hans-Jakob Riedtmann/AGZ meint, wenn es so sei, dass die Legislative heute über etwas abstimmen soll, das der Exekutive nicht einmal zur Kenntnis gebracht wurde, hat die Taskforce ihre Hausaufgaben nicht gemacht und verhält sich undemokratisch. Er plädiert klar auf Rückweisung.

Für Adrian Sury/GPK ist die ÄK Auftraggeberin und demzufolge heute als Erste zu informieren. Ein Grund, den vollumfänglichen Bericht nicht allen Delegierten zuzustellen, hing mit dem Datenschutz zusammen. Was die Mandatsverlängerung der Taskforce betrifft, werden er und Alexander Zimmer aus Interessenkonflikt diese Aufgabe nicht länger wahrnehmen können. Die GPK kann nicht gleichzeitig den ZV begleiten und kontrollieren. Er bekräftigt nochmals, dass bei der Zu- sammensetzung, die die GPK an der letzten ÄK unterbreitet hat, der Präsident der FMH wie auch das Generalsekretariat vorgesehen waren.

Pierre Vallon/Taskforce lässt den Vorwurf des mangelnden demokratischen Vorgehens nicht gelten. Die Taskforce hat anlässlich der DV vom 18.3.2015 dem ZV wie auch den DV-Delegierten einen ersten Bericht präsentiert. Diese hatten bereits wichtige Vorinformationen. Auf Wunsch der GS wurden ebenfalls die Mitarbeitenden des Generalsekretariats informiert. Die Frage stellt sich jedoch, ob ein 60-seitiger Bericht mit äusserst sensiblen und vertraulichen Informationen 200 Personen einfach so zugestellt werden kann und soll.

Jürg Schlup/Präsident FMH stellt den Ordnungsantrag, dass die Rednerliste mit dem Vertreter des Ordine abgeschlossen wird.

\section{Ordnungsantrag Schlup:}

Die Rednerliste wird nach dem Vertreter des Ordine abgeschlossen.

\section{Beschluss:}

Dem Ordnungsantrag wird mit 101 Ja und 3 Nein zugestimmt.

Josef Widler/AG meint, dass die ÄK das erhalten hat, was sie bestellt hat. Die demokratischen Rechte wurden eingehalten. Für ihn ist es richtig, dass weder der Präsident noch die GS Einsitz in der Taskforce hatten. Er stellt mündlich den Wortlaut eines noch einzureichenden Antrags vor: «Die ÄK nimmt von den durch die Taskforce vorgeschlagenen Budgetstabilisierungsmassnahmen Kenntnis. Sie geht davon aus, dass durch die vorgeschlagenen Massnahmen die Leistungen zugunsten der Mitglieder nicht geschmälert werden, und die FMH ihre Handlungsfreiheit im gewünschten Rahmen vergrössern kann. Sie erteilt dem ZV den Auftrag, erstens: die vorgeschlagenen Massnahmen zu prüfen. Zweitens: der ÄK vom 29.10.2015 einen detaillierten Massnahmenund Zeitplan vorzulegen. Drittens: an der $\ddot{K} K$ vom 29.10.2015 jene Massnahmen aufzuzeigen, die er nicht durchführen will oder kann, und die Ablehnung zu begründen. Viertens: notwendige Änderungen von Statuten, Reglementen und ÄK-Beschlüssen zu beantragen. Fünftens: die DV und die ÄK regelmässig über den Stand der Umsetzung zu orientieren. Und sie erteilt der GPK den Auftrag, der $\ddot{K} K$ regelmässig über den Fortgang der Dinge Bericht zu erstatten.»

Lars Frauchiger/VSAO bereitet das heutige Vorgehen Mühe. Einerseits verfügt er als Delegierter nicht über die vollumfänglichen Informationen und Grundlagen. Anderseits wird von ihm verlangt, innerhalb von wenigen Minuten anhand einer PowerPoint-Präsentation über Millionen zu entscheiden, die die Zukunft der FMH doch stark in die eine oder andere Richtung bewegen wird. 
Thomas Heuberger/BE stellt noch einmal klar, dass die meisten nun gehörten Voten inklusive Meinung des Rechtskonsulenten in eine ähnliche Richtung gehen. Das spricht doch eindeutig dafür, dass die Vorlage wohl Sinn machen kann, aber die Konsequenzen der einzelnen Vorschläge, die zur Diskussion kämen, eben zu wenig durchdacht und analysiert wurden. In diese Richtung äussern sich auch die Rückweisungsanträge der beiden Anträge des VSAO und des SMSR. Für ihn ist die richtige Lösung, dass der ZV als Exekutivorgan den Auftrag erhält, Vorschläge auszuarbeiten mit dem Wissen der entsprechenden Konsequenzen.

Fiorenzo Caranzano/TI findet es unverantwortlich, heute Entscheide über operationelle Tätigkeiten zu fällen. Die ÄK aber hat ein Recht zu erfahren, auf welcher Basis der ZV seine Überlegungen und Vorschläge erarbeiten wird. Es geht ihm um die Information der Ergebnisse durch die Taskforce und nicht um das Fällen von Entscheiden. Jürg Schlup/Präsident FMH schliesst mit diesem Votum die Eintretensdebatte und erteilt der Taskforce das Schlusswort.

\subsubsection{Schlussvotum Taskforce}

Peter Wiedersheim/Taskforce befremdet das heutige Vorgehen. Die Taskforce hat ihren Auftrag nach bestem Wissen und Gewissen ausgeführt und die Auftraggeberin ist nun nicht bereit, die Resultate anzuhören. Er versteht nicht, dass das Entscheidungsgremium nun die Informationen blockiert und der Taskforce nicht die Möglichkeit gibt, ihre Resultate und Schlussfolgerungen zu zeigen. Die Voten des ZV überraschen ihn, zumal er immer von einer guten Zusammenarbeit ausgegangen ist. Als Beispiel zitiert er das damalige Projekt «Führungsmodelle», wo $90 \%$ zugestimmt haben. Leider blieb es bis heute nur bei der Zustimmung und nicht bei der Umsetzung.

Seine Wunschvorstellung ist, dass jeder Departementschef als Stratege am Steuer sitzt und hinter sich ein perfektes operatives Team weiss. Den ZV sieht er in seiner Funktion überwiegend strategisch. Er wünscht sich ein Teamwork, deshalb glaubt er auch an die ÄK. Zusammen ist die FMH stark und er hofft, dass alle konstruktiv an einer FMH mitarbeiten, die es in Zukunft noch besser kann als heute. Das bleibt auch das Ziel der Taskforce. Er wünscht Klarheit darüber zu schaffen, ob die Delegierten das anhören wollen, was ein Expertengremium erarbeitet hat. Über die Verantwortung des Umsetzungsmanagements kann noch diskutiert werden.

\subsection{Abstimmungen}

Es wird folgendes Abstimmungsprozedere festgelegt:

- Antrag VSAO versus Antrag SMSR

- Obsiegender Antrag versus Eintreten
Der Antrag VSAO lautet wie folgt:

1. Nichteintreten auf die Budgetstabilisierungsvorschläge der Taskforce.

2. Auflösung der Taskforce.

3. Auftrag der $\ddot{\mathrm{AK}}$ an $\mathrm{ZV}$ und Generalsekretariat FMH, an der nächsten ÄK, nach Analyse des KPMG-Berichts begründete Vorschläge für die angestrebte Budgetstabilisierung und Effizienzgewinne zu präsentieren.

Der Antrag des SMSR lautet wie folgt:

Die ÄK nimmt vom Bericht der Taskforce Kenntnis und weist ihn an den ZV zur eingehenden Analyse mit dem Generalsekretariat zurück. Der ZV wird der ÄK vom Herbst 2015 die realisierbaren Massnahmen vorschlagen, die in das Budget 2016 integriert werden.

\section{Beschluss:}

Mit 45 Ja für den Antrag VSAO zu 89 Ja für den Antrag SMSR mit 5 Enthaltungen wird der Antrag SMRS angenommen.

Josef Widler/AGZ verlangt, dass in der nächsten Abstimmung nun über Eintreten Ja oder Nein abgestimmt werden sollte.

Thomas Heuberger/BE weist darauf hin, dass der SMSRAntrag lautet: «nimmt Kenntnis vom Bericht der Taskforce». Man kann nicht von etwas Kenntnis nehmen, was man nicht gesehen hat. Er wünscht jedoch keine Detaildiskussion mehr. Deshalb stellt er nochmals den Antrag über eine Abstimmung.

Der Wortlaut des Antrags des SMSR führt zu Unklarheiten. Jürg Schlup/Präsident FMH bittet um Präzisierung. Pierre-Alain Schneider/VD erklärt, dass der Antrag klar aussagt, dass vom Bericht Kenntnis zu nehmen sei. Der verlangte Bericht soll dem ZV zugestellt werden. Es geht nicht, dass die ÄK über etwas entscheidet, ohne die nötigen Informationen zu haben. Für ihn heisst Kenntnis nehmen, dass die Präsentation gezeigt wird. Pierre Vallon/Taskforce will von der SMSR wissen, ob hier vom vollständigen durch die KPMG erarbeiteten Bericht oder nur von der Präsentation die Rede ist.

Pierre-Alain Schneider/VD antwortet, dass hier die Präsentation, wie sie an der DV gezeigt wurde, gemeint ist. Jürg Schlup/Präsident FMH präzisiert, dass aufgrund des bereits abgestimmten Antrags Gafner die Delegierten auf eine Präsentation verzichten, wenn den Anträgen des VSAO oder der SMSR zugestimmt wird. Da dem Antrag der SMSR zwischenzeitlich zugestimmt wurde, bedeutet dies die Rückweisung an den ZV und die Unterbreitung von neuen Vorschlägen und Massnahmen an der ÄK vom 29.10.2015. Einer Präsentation wurde mit dieser Abstimmung nicht zugestimmt.

Hans-Anton Vogel/AR stellt den Rückkommensantrag, dass die Präsentation gezeigt wird. 


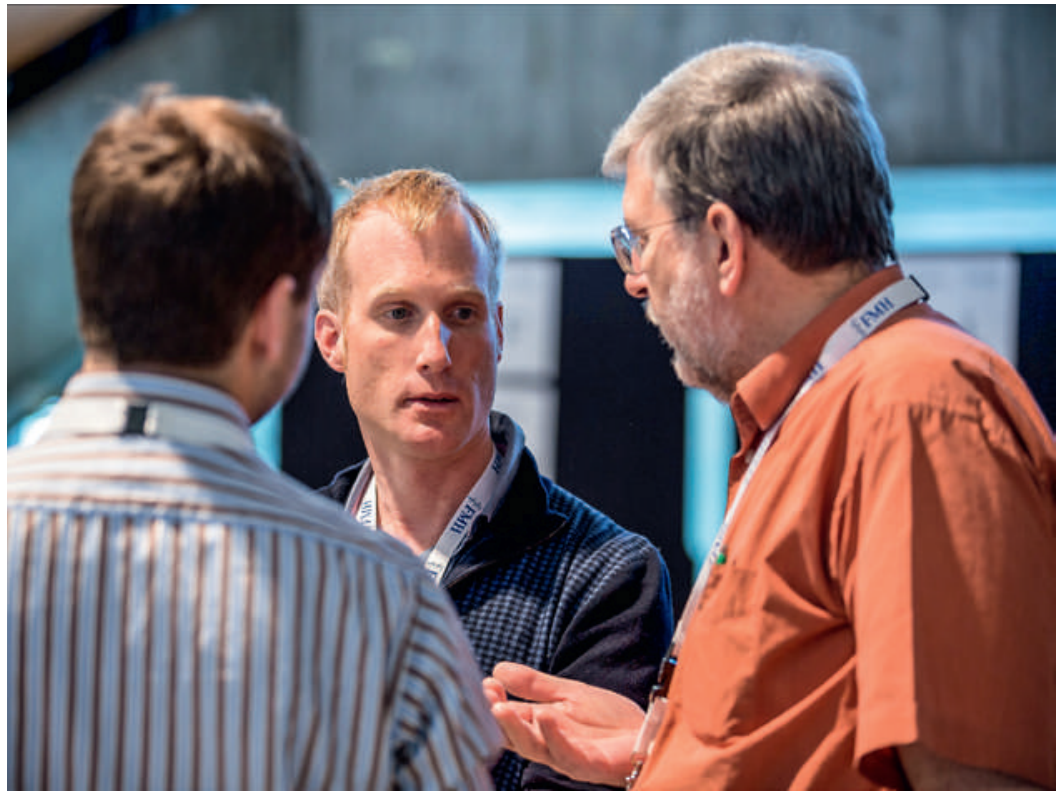

Über Generationen hinweg tauschen sich die Mitglieder der Ärztekammer aus.

Jürg Schlup/Präsident FMH empfiehlt nach einem kurzen Sitzungsunterbruch ein Vorgehen, welches vom Rechtsdienst präsentiert wird.

Hanspeter Kuhn/FMH schlägt drei Abstimmungen vor:

1. Rückkommensantrag von Hans-Anton Vogel (wir wollen Präsentation sehen)

2. Bei Gutheissung des Rückkommensantrags besteht die Möglichkeit, den Antrag des SMSR so zu splitten, dass differenziert abgestimmt werden kann. Dann gibt es die Abstimmung über die Weiterweisung an den ZV.

3. Soll die Präsentation heute auch bei Nichteintreten gezeigt werden oder nicht.

Konrad Schiess/SG findet, dass mit diesem Vorgehen das Prozedere geändert wurde. Wenn dem so ist, bringt er einen Antrag, der will, dass über die Zusatzanträge und Präsentationen erst nachher abgestimmt wird. Er schlägt eine Weiterweisung an eine Kommission - analog einer parlamentarischen Kommission - vor, welche dann der ÄK ihre Vorschläge vorlegt.

Für Hanspeter Kuhn/FMH kommt dieser Antrag zu spät. Es geht jetzt darum abzustimmen, ob auf die Detailberatung eingetreten wird oder nicht. Der SMSR beantragt die Weiterweisung an den ZV, Konrad Schiess schlägt die Weiterweisung an eine Kommission vor, wo der ZV auch vertreten ist. Wenn nun darauf eingetreten werden soll, müsste der SMSR-Antrag diesem neuen Antrag gegenübergestellt werden. Der obsiegende Antrag würde dann übernommen.

Michaël Hagmann/VD schlägt vor, die Abstimmung, die Präsentation in jedem Falle, ob Eintreten oder nicht, vorzuziehen.
Jürg Schlup/Präsident FMH ist mit diesem Vorgehen einverstanden. Es wird nun über die Anträge abgestimmt:

Rückkommensantrag Vogel:

Der Antrag lautet: Wir wollen die Präsentation sehen.

\section{Beschluss:}

Der Rückkommensantrag wird mit 117 Ja, 21 Nein und 1 Enthaltung gutgeheissen.

\section{Antrag:}

Wir wollen die Präsentation in jedem Fall, ob Eintreten oder nicht, sehen.

Beschluss:

Der Antrag wird mit 110 Ja, 20 Nein und 1 Einhaltung gutgeheissen.

\section{Antrag SMSR:}

Keine Detailberatung und Weiterweisung an ZV gemäss Antrag SMSR und Nichteintreten.

\section{Beschluss:}

Dieser Antrag wird mit 107 Ja, 25 Nein und 4 Enthaltungen gutgeheissen.

Aufgrund dieser Abstimmungsergebnisse erübrigt sich die Detailberatung. Die KPMG jedoch wird ihren Bericht präsentieren. Mit der Annahme des Antrags der SMSR ist auch die Frage des Mandats der Taskforce geklärt. Das Geschäft wird an den ZV überwiesen.

Stefan Greuter/VSAO stellt den Ordnungsantrag, die Entscheidtraktanden vor der Präsentation der KPMG vorzuziehen.

\section{Ordnungsantrag Greuter/VSAO}

Bevor die Präsentation gezeigt wird, sind die Traktanden 10 und 11 zu beraten.

\section{Beschluss:}

Dem Ordnungsantrag Greuter wird mit $103 \mathrm{Ja}$, 5 Nein und 12 Enthaltungen zugestimmt.

Pierre Vallon/Taskforce wünscht, sein Taskforce-Mandat heute zu beenden. Unter den gegebenen Umständen will er seine Arbeit nicht mehr weiterführen.

\section{Gesamtrevision TARMED}

Die Gesamtrevision TARMED war ein grosses Projekt, das Ernst in den letzten Jahren mit grosser Akribie und sehr viel Engagement geführt und betreut hat. Das Projekt ist auf der Zielgeraden und die Verteilungskämpfe beginnen. Urs Stoffel/ZV FMH, der dieses Dossier von Ernst übernommen hat, dankt an dieser Stelle dem Team «Ambulante Tarife» in Olten, das mit einem ausserordentlichen Einsatz im Sinne der Ärzteschaft unermüdlich an der Revision arbeitet.

Das Projekt «Gesamtrevision TARMED» ist so weit fortgeschritten, dass eine FMH-interne Vernehmlassung von einzelnen Kapiteln auf tariftechnischer Ebene ge- 
startet werden kann. Bei dem nun präsentierten Vernehmlassungsprozess geht es um den Ablauf der Vernehmlassung auf Stufe Fachteam und dem geplanten Prozess FMH intern der Ergebnisse aus den Fachteams. Alle Beteiligten sollten die Möglichkeit haben, offen und transparent zu sehen, was sich und wie es sich verändert hat, und dazu Stellung zu nehmen.

\section{Prozess 1: Ebene Fachteam}

Das Fachteam hat den Auftrag für die Aktualisierung der Nomenklatur (medizinischer Leistungskatalog) und gegebenenfalls der Infrastruktur. Der Output dieser Fachteamarbeit ist der Leistungskatalog inkl. Minutage wie Inputs zur Infrastruktur. Sind die beteiligten Fachgesellschaften mit den Vorschlägen der Revisionspartner nicht einverstanden, gibt es zwei Möglichkeiten. Das Fachteam, das jeweils von einem Vertreter H+, FMH oder MTK (Revisionspartner) geleitet wird, hat den Auftrag, einen gemeinsamen Vorschlag an die Technische Kommission (TeKo) der Revisionspartner weiterzuleiten. In den Fachteams sind neben den betroffenen Fachgesellschaften, die in diesem Kapitel im Vergleich am meisten abrechnen, auch Tarifexperten der Revisionspartner $\mathrm{H}+$, FMH und MTK vertreten.

Was passiert bei Uneinigkeit zwischen den Revisionspartnern: Das heisst, die FMH-Vertreter (Fachgesellschaften und Tarifexperten) sind sich einig, aber vertreten einen anderen Tarifierungsvorschlag als beispielsweise H+ oder MTK. In diesem Fall sind die Eskalationsstufen im Rahmen des Revisionsprojekts klar geregelt. Zunächst in der operativen Leitung der TeKo und im zweiten Schritt in der Steuerungskommission. In beiden Gremien sind wiederum alle Revisionspartner vertreten und die Entscheidungswege sind vorgegeben.

Was passiert bei Uneinigkeit innerhalb der FMH: Um einen gemeinsamen Vorschlag einzureichen ist Voraussetzung, dass die Vertreter der einzelnen Revisionspartner (FMH, MTK, H+) sich einig sind. Wenn es FMHintern zwei oder mehrere Vorschläge gibt, sei es durch zwei Fachgesellschaften, oder auch wenn die Vertreter der Fachgesellschaften und die Tarifexperten der FMH sich auf keinen gemeinsamen Vorschlag einigen können, muss der Prozess definiert werden, welchen Vorschlag die FMH im Fachteam vertritt bzw. einbringt. Der ZV FMH ist in diesen Belangen das Eskalationsund Entscheidungsgremium.

Wenn man sich im Fachteam nicht einig ist, gibt es die FMH-interne Möglichkeit, wo die Vorschläge dem ZV FMH vorgelegt werden oder auch die Ebene der Revisionspartner. Falls sich hier Differenzen ergeben, sind die TeKo und die Steuerungskommission für eine Einigung zuständig. Letztlich gibt es dann die Freigabe und den Revisionsvorschlag durch das Fachteam.
Prozess 2: Externe Vernehmlassung (Revisionspartner) Nach Freigabe durch das Fachteam wird der Vorschlag durch die TeKo geprüft und genehmigt.

Prozess 3: Interne Vernehmlassung FMH

Die Fachgesellschaften werden über die Resultate informiert und der Browser aufgeschaltet. Alle Fachgesellschaften haben die Möglichkeit, die revidierten Kapitel anzusehen und zu prüfen, ob die vorgelegte Tarifstruktur den Tarifierungsgrundsätzen entspricht und ihren medizinischen Alltag abbildet.

Die Tarifdelegierten bzw. das Präsidium einer Fachgesellschaft sammeln nun die Feedbacks über ein publiziertes Kapitel und leiten sie der FMH weiter, welche wiederum diese zur Durchsicht an die zuständige Fachgesellschaft weitergibt, die den Vorschlag miterarbeitet hat. Die Art des Feedbacks umfasst die Gründe der Beanstandungen, Meldung fehlender Leistungen wie auch Alternativvorschläge mit Belegen.

Falls kein Handlungsbedarf besteht, ist der Prozess 3 beendet. Bei Uneinigkeit entscheidet der ZV FMH und leitet die Eingaben zur Prüfung an die TeKo weiter. Sollte es auf dieser Ebene keine Lösung geben, entscheidet die Steuerungskommission und die Evaluationsphase ist abgeschlossen.

Die einzelnen Eingaben im Rahmen des Vernehmlassungsprozesses FMH intern werden nicht einzeln beantwortet, sondern Antworten, Gründe und Entscheidungen für alle beteiligten Organisationen publiziert und kommuniziert.

Prozess 4: Konsolidierung / Abschlussarbeiten Nach Abschluss des Prozesses 3 wird der Browser aktualisiert. Allfällige Anpassungen (tariftechnischer Natur) können nach wie vor einfliessen. Die technische Strukturrevision ist somit abgeschlossen und das Going public der technischen Struktur wird freigegeben. Dieses Vorgehen wurde sowohl im ZV, in der DV wie auch am Tarifdelegiertentag vorgestellt und besprochen.

Als Nächstes werden die Tarifdelegierten und Präsidenten über den Start des Prozesses 3 in der Vernehmlassung informiert. Ziel ist es, an der ÄK vom 29.10.2015 die Rahmenverträge sowie die vollständige Tarifstruktur zu genehmigen.

Michel Matter/GE will wissen, ob die Frage der Kostenneutralität wiederum im Raum steht, wenn es einen Modellwechsel geben sollte. Gemäss aktuell gültigem Vertrag ist dies so vorgesehen.

Urs Stoffel/ZV FMH informiert, dass diese Frage am Tarif-Delegiertentag vom 6.5.2015 ein Hauptthema war. Was gestern vorgestellt wurde, hat gezeigt, dass die Revision einer Tarifstruktur, solange man das Modell, das dahinter steht, nicht ändert, eben eine Revision und kein Wechsel des Tarifmodells ist. Am Modell wurde für die Revision der Tarifstruktur nichts verän- 
dert. Deshalb ist Art. 59c Abs. 1c der Krankenversicherungsverordnung - und das ist die einzige rechtliche Grundlage, die diese Kostenneutralität fordert - aus Sicht FMH klar nicht erfüllt. Die FMH wird diese Haltung auch weiterhin so vertreten.

Philippe Rheiner/GE hat zum Prozess 2 eine Frage. Was passiert, wenn die technische Kommission den Vorschlag nicht genehmigt? Geht es dann zurück an die Fachgesellschaft, die entscheidet?

Urs Stoffel/ZV FMH erklärt, dass die Fachgesellschaft vor einem abschliessenden Entscheid nochmals angehört wird.

Die Delegierten stimmen über die zwei Anträge separat ab.

Antrag:

Genehmigung der technischen Vernehmlassungsprozesse der Tarifstruktur gemäss Ziffer 2 des Rundschreibens.

Beschluss:

Der Antrag wird mit 153 Ja mit 4 Enthaltungen angenommen.

\section{Antrag:}

ZV FMH als Eskalations- und Entscheidungsorgan FMH intern.

Beschluss:

Der Antrag wird mit 145 Ja zu 1 Nein und 7 Enthaltungen gutgeheissen.

\section{Bericht zu HPC}

Am 30.6.2015 endet die Zusammenarbeit der FMH mit der Swisscom als Provider für die Herausgabe der HPC. Swisscom bietet ab 2016 keinen kartengestützten Zertifikatsservice mehr an. Urs Stoffel/ZV FMH informiert, dass der ZV diese Gelegenheit genutzt hat, die Strategie zu überdenken, auch bezüglich elektronischer Identitäten (eID). Aufgrund von Umfragen (eHealth-Barometer) und den Erfahrungen bei eHealth-Pilotprojekten («Mon Dossier Medical» in GE) liegt die Zukunft nicht in der eID auf Karten als Medium. Die FMH setzt künftig auf eine Multichannel-Strategie. Im Projekt HPC 2.0 wurden vier Umbaustufen entwickelt, wovon der ZV die Umbaustufen 1 und 2 bereits bewilligt hat. Diese Umbaustufen umfassen die Ablösung der HPC vom Provider der Swisscom per 30.6.2015 und die Übernahme durch den FMH-eigenen Provider HIN zusammen mit QuoVadis ab 1.7.2015. Der Betrieb der bestehenden HPCKarten ist nicht gefährdet. In einer zweiten Phase (Umbaustufe 3 und 4) soll 2016 der Ausbau der nicht kartengestützten eID durch HIN mittels Client Lösung auf dem PC, Hintergrund der eID auf Hochsicherheits-Servern und Zugriff auf eID via «Cloud» erfolgen.

QuoVadis, eine von vier Unternehmungen, die Zertifi- kate herausgeben können, wurde als neuer Provider bestimmt. Mit dem Wechsel vom kartenbasierten Zertifikat zur neuen Multichannel-Strategie werden mittel- und langfristig die Betriebskosten der HPC deutlich gesenkt werden. Wegen der komplexen technischen Anforderungen beim Providerwechsel ist eine Verzögerung von ein bis zwei Monaten bei Neubestellungen von HPC-Karten mit Zertifikat auf der Karte möglich. Diejenigen Zertifikate, die im Umlauf sind und funktionieren, sind nicht gefährdet. Aber bei neuen Zertifikaten kann es sein, dass diese erst Ende August dieses Jahres funktionieren werden.

Es gibt keine Wortmeldungen und die Delegierten nehmen diese Informationen zur Kenntnis.

\section{IPI}

\subsection{Aktivitäten 2015}

Anne-Geneviève Bütikofer/GS FMH informiert über die aktuelle Vertragssituation und den Stand der Aktivitäten 2015. Nach der Genehmigung des Sonderbeitrags in der ÄK Oktober 2013 ist der Leistungsvertrag im Februar 2014 unterzeichnet worden. Die ÄK hat am 30.10.2014 der Verlängerung des Leistungsvertrages nur um ein Jahr zugestimmt. Ein Nachtrag 2015 zum Leistungsvertrag 2014 zwischen dem IPI und der FMH ist bis heute noch nicht abgeschlossen, da die Anhänge (Bestandteil des Nachtrages) nach wie vor ausstehend sind. Trotz mehrmaliger Intervention fehlt u.a. die Balance Scorecard. Im Vergleich zu 2014, wo das IPI 17 Projekte geplant hat, sind für 2015 nur fünf Projekte vorgesehen. Der angepasste Businessplan 2015-2017 und der Finanzplan 2015 liegen mittlerweile vor.

Die Arbeitsgruppe eHealth ist über die aktuelle Situation informiert und unterstützt das Vorgehen des ZV, die noch ausstehenden Unterlagen einzufordern. Fazit ist, dass die FMH heute keine Vertragsbeziehung mit dem IPI hat. Der a.o. Sonderbeitrag ist zwar fakturiert, eine Zahlung ans IPI kann jedoch erst erfolgen, wenn alle Unterlagen vollständig beim Generalsekretariat FMH eingereicht sind. Über weitere Aktivitäten kann heute nicht berichtet werden.

Hans-Anton Vogel/VEDAG bittet, bezüglich der geforderten Administration Augenmass zu behalten. Das IPI verfügt über zu wenig Personal, um einen Grossteil ihrer Ressourcen dafür aufzuwenden. Der Geschäftsführer macht eine sehr gute Arbeit und dies sollte honoriert werden. Das IPI hat für die Zukunft eine äusserst wichtige Aufgabe, die nicht durch eine unsägliche Administration zum Scheitern verurteilt werden sollte. Anne-Geneviève Bütikofer/GS FMH nimmt diese Bemerkungen ernst und weist darauf hin, dass die fehlenden 
Unterlagen auch für das Generalsekretariat einen grossen Mehraufwand zur Folge hat. Das Generalsekretariat hat die Aufgabe und Pflicht, die Erfüllung des Leistungsvertrags wie den Mitteleinsatz zu prüfen. Aus operativer Sicht ist dies zur Zeit nicht möglich, da wichtige Unterlagen ausstehend sind. Wenn die Organe entscheiden, die Aktivitäten des IPI nur unter dem politischen Aspekt weiterzuführen, ist dies eine politische, jedoch nicht eine operative Entscheidung.

Jean-Pierre Pavillon/VD möchte wissen, ob das IPI bis heute eine konkrete nutzbare Leistung erbracht hat.

Anne-Geneviève Bütikofer/GS FMH bestätigt die positive Zusammenarbeit, meint jedoch, dass zu einer guten Unternehmungsführung auch die Erledigung der administrativen Aufgaben gehört.

Daniel Schröpfer/VSAO bekräftigt, dass der VSAO die Projekte des IPI unterstützt. Heute hat die ÄK abgestimmt, dass für Leistungen über CHF 10000 Leistungsverträge abzuschliessen sind. Und dafür ist eine Gegenleistung gefordert. Er fragt sich, wer in der freien Wirtschaft ohne Vorlage einer Balance Scorecard knapp CHF 1 Mio. pro Jahr erhält.

Für Anne-Geneviève Bütikofer/GS FMH ist eine Nachverfolgung der Arbeiten für einen solch grossen Betrag unabdingbar. Die Balance Scorecard ist ein wichtiges Instrument, um die Ziele und Erreichung der Meilensteine zu überprüfen. Das Generalsekretariat benötigt dieses Dokument, um nachvollziehen zu können, wofür das gesprochene Geld verwendet wurde.

Hans-Anton Vogel/VEDAG erinnert an den Entscheid der ÄK, das IPI in das Departement eHealth zu integrieren. Er sieht jedoch von dieser Integration noch wenig Konkretes. Dies müsste doch die erste Aufgabe sein und nicht der Abschluss dieser Verträge. Er bittet nochmals, das IPI zu unterstützen.

Anne-Geneviève Bütikofer/GS FMH versteht das Anliegen, hält jedoch fest, dass es sich hier um zwei verschiedene Dinge handelt. Einerseits geht es um die Aktivitäten des IPI für das Jahr 2015. Anderseits ist die Integration in das Departement eHealth für 2016 geplant. Das Generalsekretariat fühlt sich gegenüber der ÄK für die Rapportierung verpflichtet.

Gerhard Schilling/Präsident IPI nimmt zu den fehlenden Unterlagen Stellung. Er informiert die Delegierten über die effektiven Arbeiten des Geschäftsführers, der alleine für die Erledigung der Erstellung der administrativen Unterlagen 40\% seiner Arbeitszeit aufwenden muss. Er rapportiert über den Stand der aktuellen Projekte und die Durchführung der Going-Paperless-Kurse. Das IPI sei im Moment an vielen Projekten beteiligt und kann entsprechend auch einen Erfolg ausweisen. Er bittet die Delegierten, diese Arbeit zu honorieren und das IPI nicht mit Nebensächlichkeiten zu belasten.
Für Daniel Schröpfer/VSAO ist die Balance Scorecard keine Nebensächlichkeit. Noch einmal: Wer erhält heute einen Kredit von CHF 1 Mio., ohne etwas vorzuweisen?

Andreas Häfeli/AGZ hatte bereits bei der heutigen Abstimmung über die Leistungsverträge, vor allem betreffend Unterstellung der Leistungsverträge, ein ungutes Gefühl. Da er zu diesem Zeitpunkt das Wort nicht ergriffen hat, bittet er an dieser Stelle den ZV, das Flussdiagramm und die Vorgehensweise zu überprüfen. Es kann nicht sein, dass aus formalistischen Gründen der Vertragsabschluss auf Stufe Generalsekretariat blockiert werden kann.

Anne-Geneviève Bütikofer/GS FMH möchte dem IPI keine Steine in den Weg legen. Sie erinnert jedoch daran, dass sie als GS die Verantwortung über die Budgeteingaben habe. Und angesichts der aktuellen finanziellen Situation bei der FMH ist ein bisschen Formalismus und Strenge notwendig geworden.

\subsection{Integration IPI in das Departement eHealth der FMH}

Zurückkommend auf die vorherige Diskussion unterstützt Urs Stoffel/ZV FMH sowohl das IPI in seinen Bestrebungen wie auch die GS FMH, auf die ausstehenden Dokumente zu beharren, um einen neuen Leistungsvertrag abzuschliessen. Was die Integration des IPI in das Departement eHealth betrifft, hat die Arbeitsgruppe eHealth, bei der auch das IPI vertreten ist, zwischenzeitlich verschiedene Modelle diskutiert und existierende Modelle geprüft. Das Departement eHealth schlägt ein Modell analog zum Modell SAQM vor, das so übernommen werden könnte. Die Organisation soll möglichst schlanke Strukturen haben, Synergien nutzen und Doppelspurigkeiten vermeiden. Das Advisory Board IPI ist ein Input-Gefäss, in dem auch die Erfahrungen der IPI-Gründer einfliessen. Das IPI bleibt in der bisherigen Form nicht bestehen. Der neue Name soll auf einen Neuanfang hinweisen. Das Leitungsgremium, wie hier präsentiert, wurde von der Arbeitsgruppe eHealth begrüsst. Das Modell wird nun weiterentwickelt, weshalb die Arbeitsgruppe heute von der ÄK die Zustimmung einholen möchte, um auch die Integration IPI in das Departement eHealth voranzutreiben.

Daniel Schröpfer/VSAO will wissen, weshalb es noch ein Zentrum für Praxisinformatik braucht, wenn es unter der Leitung eHealth FMH läuft? Dieser Begriff ist eher verwirrlich

Urs Stoffel/ZV FMH erklärt, dass dieser Vorschlag ein «work in progress» sei und der ÄK im Herbst das abschliessende Organigramm mit den definitiven Bezeichnungen präsentiert wird.

Jürg Nadig/SGMO weist bei den präsentierten Modellen 
auf einen Unterschied hin. Seiner Meinung nach sind bei der SAQM alle Fachgesellschaften vertreten, in der Arbeitsgruppe eHealth jedoch nur die Dachverbände. Für ihn stellt sich die Frage, ob sich die Fachgesellschaften in diesem Modell genügend einbringen können. Urs Stoffel/ZV FMH hält fest, dass die Arbeitsgruppe eHealth nicht allein die Dachverbände, sondern auch die Fachgesellschaften umfasst. Falls nur der Dachverband vertreten sein sollte, kann selbstverständlich auch die Basis Einsitz nehmen.

Zur Frage der Finanzierung erläutert Urs Stoffel/ZV $F M H$, dass bis Ende 2015 ein Sonderbeitrag gesprochen ist. Bei der Integration in das Departement eHealth handelt es sich um einen neuen Auftrag bzw. eine neue Aufgabe. Das Departement eHealth schlägt entweder die Budgetierung der neuen Aufgaben und die Erhöhung des regulären Budgets oder die Erhöhung des ordentlichen Mitgliederbeitrags statt eines Sonderbeitrags vor.

Adrian Sury/Präsident GPK ermahnt, dass die GPK bereits mehrfach dargelegt hat, wiederkehrende Aufwendungen nicht über einen Sonderbeitrag zu finanzieren. Die Sonderbeiträge sind für ausserordentliche Zwecke. Er macht beliebt, dass man den zweckgebundenen Sonderbeitrag aus dem Vorschlag streicht.

Hanspeter Kuhn/FMH weist auf die Regelung der Sonderbeiträge, die in Art. 11 Abs. 3 der Statuten wie folgt geregelt sind:

${ }^{3}$ Die FMH und die KG setzen unter Beachtung des Kostenverursacherprinzips folgende Mitgliederbeiträge fest: - den allgemeinen jährlichen Grundbeitrag, allfällige Sonderbeiträge für bestimmte Mitgliedergruppen oder für bestimmte Projekte. Die Höhe des Grundbeitrags bemisst sich nach der beruflichen Stellung des Mitglieds. Die GO regelt die diesbezüglichen Kategorien sowie Kriterien für Beitragsreduktionen.

Daniel Schröpfer/VSAO weiss nicht, warum man nun unter Punkt 2 einen Entscheid über die Finanzierung fällen soll. Dieser Entscheid wäre ja eigentlich erst im Oktober 2015 geplant.

Für Urs Stoffel/ZV FMH bedeutet ein Entscheid, dass die Delegierten einverstanden sind, die zwei Finanzierungsmöglichkeiten zu prüfen. Wenn die Delegierten sich nur für eine Variante aussprechen, wird nur diese weiterverfolgt.

Hans-Jakob Riedtmann/AGZ erinnert daran, dass vor diesem Traktandum bei der Budgetstabilisierung davon gesprochen wurde, den operativen Spielraum festzulegen. Der ZV wird im Oktober 2015 der ÄK vorlegen, welche personellen und finanziellen Ressourcen für eben einen solchen operativen Spielraum frei werden. Wenn den Delegierten das IPI wirklich am Herzen liegt, kann eine Priorisierung mit den frei werdenden
Mitteln beschlossen werden. Es braucht somit keine ordentliche Mitgliederbeitragserhöhung.

Es wird wie folgt über die beiden Anträge abgestimmt:

\section{Antrag ZV:}

Weiterverfolgung des vorgeschlagenen Modells analog SAQM zur Integration des IPI in das Departement eHealth und damit in die Strukturen der FMH gemäss Auftrag der ÄK vom 30.10.2014.

\section{Beschluss:}

Dem Antrag wird mit 104 Ja, 9 Nein und 11 Enthaltungen zugestimmt.

\section{Antrag ZV:}

Entscheid, ob zur Finanzierung des zusätzlichen Auftrags IPI ein zweckgebundener Sonderbeitrag oder eine Erhöhung des ordentlichen Mitgliederbeitrags vorgesehen werden soll.

\section{Beschluss:}

Der Antrag wird mit 32 Ja, 57 Nein und 25 Enthaltungen abgelehnt.

Gemäss Urs Stoffel/ZV FMH ist mit diesem Entscheid die Finanzierung über das ordentliche Budget, ohne Erhöhung des Budgets und ohne Sonderbeitrag oder Erhöhung des Mitgliederbeitrags, umzusetzen.

\section{Präsentation Bericht Taskforce durch die KPMG}

Wie unter Traktandum 8 entschieden, erhält Dr. Walter Gratzer/KPMG die Gelegenheit, den Ergebnisbericht der Taskforce vorzustellen.

Gemäss Auftrag und Beschluss der ÄK hat die Taskforce der KPMG den Auftrag für die Basisanalyse erteilt. Als Erstes wurde jede einzelne Dienstleistung der FMH angeschaut, bewertet und beurteilt. Als Zweites wurde mit 24 Personen der FMH Interviews geführt, d.h. mit allen ZV-Mitgliedern, der GS sowie allen Kadermitarbeitenden. Dort wo sinnvoll und zielführend, wurden auch Benchmark-Vergleiche mit externen Dritten gemacht, z.B. im IT-Bereich. Daraus resultierend wurden entsprechende Massnahmenpakete und -potentiale zusammengestellt. Eine Präsentation des Berichts fand in der DV, beim ZV wie auch bei den Mitarbeitenden der FMH statt. Der Taskforce war wichtig, sehr detailliert und differenziert die Dinge zu analysieren, sowohl in den Abteilungen wie in den Themen. Für die Vorgabe, einen operativen Spielraum im Rahmen von CHF 4-6 Mio. zu erreichen, wurden nach dieser Analyse Potentiale ermittelt. Ebenso wurden Quick-Wins Dinge, die man schnell realisieren kann - identifiziert. Es wird kein Stellenabbau vorgesehen. Der Ansatz zielt auf eine Umschichtung der Personalressourcen, nämlich Freiräume für neue Projekte und neue Initiativen zu schaffen, die die FMH als Ganzes stärken. 
Eine Ergebnisverbesserung kann immer nur über zwei Hebel erfolgen, einerseits durch Ertragssteigerungen und andererseits über Aufwandreduktionen. Im Bereich der Ertragssteigerungen gibt es die Möglichkeit, höhere Erträge zu erwirtschaften, bessere Margen $\mathrm{zu}$ realisieren oder Zusatzerträge zu generieren. Hier konnten keine substantiellen Potentiale ermittelt werden. Von daher fokussiert sich das gesamte Thema der Ertragssteigerung ausschliesslich auf höhere Erträge, nämlich auf die Erhöhung der Mitgliederbeiträge. Bei der Reduktion des Aufwands, also der Sach- und Personalkosten, konnten Einsparpotentiale erörtert werden. Dr. Walter Gratzer/KPMG stellt diese eruierten Potentiale vor, die sich vor allem auf die Anpassung des Dienstleistungsportfolios, die Optimierung des Projektportfolios, die Prozessoptimierung sowie die Optimierung In-/Outsourcing beziehen. Die Umsetzung der Massnahmen wäre grundsätzlich ohne Stellenabbau realisierbar gewesen, da die Umsetzung in einem Zeitrahmen von 3,5 Jahren zu erfolgen hätte. Eine Redimensionierung des ZV, die Bildung von sechs Kompetenzzentren, übergreifende Massnahmen wie Übernahme der Sitzungsentschädigungen der ÄK-Mitglieder durch delegierende Gesellschaften, Optimierung der HPC durch Wechsel des Servicepartners, Reduktion der jährlichen DV von sechs auf fünf und die Aufhebung des Standorts in Olten sind weitere prüfenswerte Vorschläge.

Da der ZV nun für die weiteren Arbeiten zuständig ist, sind die Präsentation des Umsetzungsmanagements wie die Erläuterung der Entscheidungsanträge obsolet geworden. Es ist nun Aufgabe des ZV und der GS, das Umsetzungsmanagement festzulegen. Die KPMG hat als externe Beraterfirma einen externen Blick auf die Dinge geworfen und natürlich auch den internen Blick in ihren Bericht integriert. Dr. Walter Gratzer/KPMG hält fest, dass es in der FMH wie auch in jeder anderen Organisation unterschiedliche Interessenlagen und Meinungen gibt, was unterschiedliche Ansätze zur Realisierung der einen oder anderen Massnahme zur Folge haben wird. Er gibt als Empfehlung mit auf den Weg, einen sauberen und geordneten Prozess aufzusetzen, damit die Dinge sich auch im Sinne eines konstruktiven Interessenausgleichs entwickeln und am
Ende substantielle Vorschläge auf dem Tisch sind. Er dankt für die Möglichkeit der Präsentation und die gute Zusammenarbeit.

Jürg Schlup/Präsident FMH dankt Dr. Walter Gratzer für seine Ausführungen. Er ist überzeugt, dass der ZV auf der Basis dieses Berichts der ÄK im Herbst umsetzbare Massnahmen vorschlagen wird. Er dankt an dieser Stelle ebenfalls den Mitgliedern der Taskforce, vor allem Peter Wiedersheim und Adrian Sury, für die wertvolle Arbeit, welche mit einer grossen Zusatzbelastung verbunden war.

\section{Informationen aus Zentralvorstand, SIWF, Generalsekretariat und Abteilungen}

\subsection{Markenpflege: Überarbeitung Logos}

Die Delegierten haben zu diesem Geschäft eine Sitzungsunterlage erhalten. Es gibt weder Fragen noch Wortmeldungen.

\subsection{Fragen an die Mitglieder des Zentral- vorstandes}

Es gibt keine weiteren Fragen an die Mitglieder des ZV.

\section{Varia}

\subsection{FMH-Sitzungsplan 2016}

Die Delegierten nehmen die Sitzungsdaten 2016, welche im ZV und in der DV bereits beschlossen wurden, zur Kenntnis. Sollten sich Grossveranstaltungen mit bereits festgelegten Daten im 2016 kreuzen, bittet die Generalsekretärin um entsprechende Mitteilung an das Zentrale Sekretariat. Die ÄK finden im nächsten Jahr am 26. Mai und 27. Oktober 2016 statt.

Die Ärztekammer wird pünktlich beendet. Jürg Schlup dankt allen Delegierten für das engagierte und konstruktive Mitwirken und wünscht eine gute Heimkehr. Er informiert, dass aufgrund aller beschlossenen Traktanden die geplante ausserordentliche ÄK vom 3.6.2015 nicht durchgeführt wird. Die nächste Ärztekammer findet somit am 29. Oktober 2015 statt. 


\begin{tabular}{ll} 
GlOSSar & \\
FMH & Verbindung der Schweizer Ärztinnen und Ärzte \\
FMPP & Foederatio Medicorum Psychiatricorum et Psychotherapeuticorum \\
GPK & Geschäftsprüfungskommission \\
GO & Geschäftsordnung \\
GS & Generalsekretärin \\
H+ & Vereinigung schweizerischer Krankenhäuser \\
HIN & Health Info Net AG \\
HPC & Health Professional Card \\
IPI & Institut für Praxisinformatik \\
KKJPD & Konferenz der Kantonalen Justiz- und Polizeidirektorinnen und -direktoren \\
MedBG & Medizinalberufegesetz \\
MFE & Berufsverband der Haus- und Kinderärztinnen Schweiz \\
MTK & Medizinaltarif-Kommission UVG \\
NKVF & Nationale Kommission zur Verhütung von Folter \\
RA & Rechtsanwalt/Rechtsanwältin \\
SAMW & Schweizerische Akademie der Medizinischen Wissenschaften \\
SAOM & Schweizerische Akademie für Qualität in der Medizin \\
SÄZ & Schweizerische Ärztezeitung \\
SFSM & Swiss Federation of Specialities in Medicine \\
SGIM & Schweizerische Gesellschaft für Allgemeine Innere Medizin \\
SGMO & Schweizerische Gesellschaft für Medizinische Onkologie \\
SGU & Schweizerische Gesellschaft für Urologie \\
SMSR & Société médicale de la Suisse romande \\
SIWF & Schweizerisches Institut für ärztliche Weiter- und Fortbildung \\
TARMED & Tarif Medizin für ambulante Einzelleistungen \\
TeKo & Technische Kommission \\
VEDAG & Verband deutschschweizerischer Ärztegesellschaften \\
VLSS & Verein Leitende Spitalärzte Schweiz \\
VSAO & Verband Schweizerischer Assistenz- und Oberärztinnen und -ärzte \\
VwVG & Bundesgesetz über das Verwaltungsverfahren \\
ZGB & Schweizerisches Zivilgesetzbuch \\
ZPO & Schweizerische Zivilprozessordnung \\
ZV & Zentralvorstand \\
\hline
\end{tabular}

